\title{
Asymmetric Total Synthesis of TAN-1085 Facilitated by Pd-Catalyzed Atroposelective C-H Olefination
}

Jun Fan, Qi-Jun Yao, Yan-Hua Liu, Gang Liao, Shuo Zhang, Bing-Feng Shi*

Department of Chemistry, Zhejiang University, Hangzhou 310027, China

E-mail: bfshi@zju.edu.cn

\section{Content}

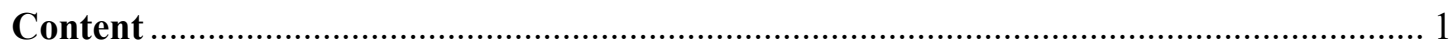

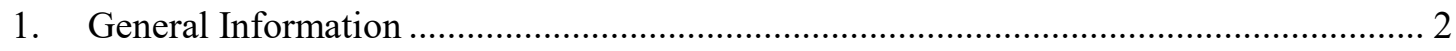

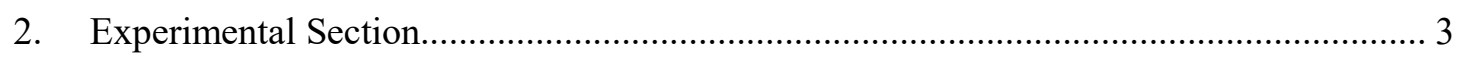

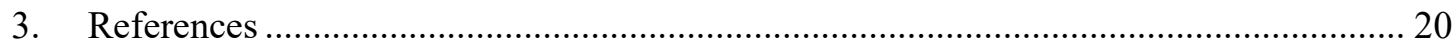

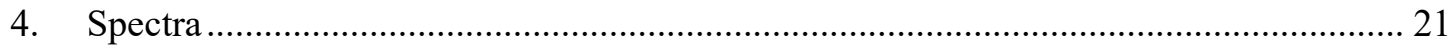




\section{General Information}

All reactions were carried out under $\mathrm{N}_{2}$ or Ar atmosphere with dry solvents under anhydrous conditions, unless otherwise noted. Anhydrous acetonitrile $\left(\mathrm{CH}_{3} \mathrm{CN}\right)$, methylene chloride $\left(\mathrm{CH}_{2} \mathrm{Cl}_{2}\right)$ were distilled from calcium hydride prior to use. Tetrahydrofuran (THF) was distilled from sodium-benzophenone prior to use. Triethylamine $\left(\mathrm{Et}_{3} \mathrm{~N}\right)$, 1,1,1,3,3,3-hexafluoro-2-propanol (HFIP), ethanol (EtOH), acetone, $N, N$-dimethylformamide (DMF), 1,2-dimethoxyethane (DME), ethyl acetate (EtOAc), acetic acid $(\mathrm{AcOH})$ and petroleum ether were purchased commercially and were used without further purification, unless otherwise stated. Reagents were purchased commercially and were used without further purification. NMR spectra were recorded on a Bruke NMR DRX-400 spectrometer operating for ${ }^{1} \mathrm{H}$ NMR at $400 \mathrm{MHz}$, ${ }^{13} \mathrm{C} \mathrm{NMR}$ at $100 \mathrm{MHz}$, Chemical shifts were given relative to TMS (0.00 ppm), $\mathrm{CDCl}_{3}$ (7.26 ppm for ${ }^{1} \mathrm{H}$ NMR, 77.16 ppm for ${ }^{13} \mathrm{C}$ NMR), DMSO (2.50 ppm for ${ }^{1} \mathrm{H}$ NMR, $39.52 \mathrm{ppm}$ for $\left.{ }^{13} \mathrm{C} \mathrm{NMR}\right)$. The following abbreviations were used to describe peak splitting patterns when appropriate: $\mathrm{br}=$ broad, $\mathrm{s}=$ singlet, $\mathrm{d}=$ doublet, $\mathrm{t}=$ triplet, $\mathrm{q}=$ quartet, $\mathrm{dd}=$ doublet of doublet, $\mathrm{m}=$ multiplet. Coupling constants, $J$, were reported in hertz $(\mathrm{Hz})$. Optical rotations were measured using a $1 \mathrm{~mL}$ cell with a $1 \mathrm{dm}$ path length on Perkin Elmer 341 at $589 \mathrm{~nm}$ at $20^{\circ} \mathrm{C}$. Mass spectroscopy data of the products were collected on an HRMS-TOF instrument using ESI ionization. HPLC analyses were performed on Shimadzu instrument using a chiral stationary phase column (Daicel Co. CHIRALPAK). 


\section{Experimental Section}

\section{Synthesis of $7^{1}$}

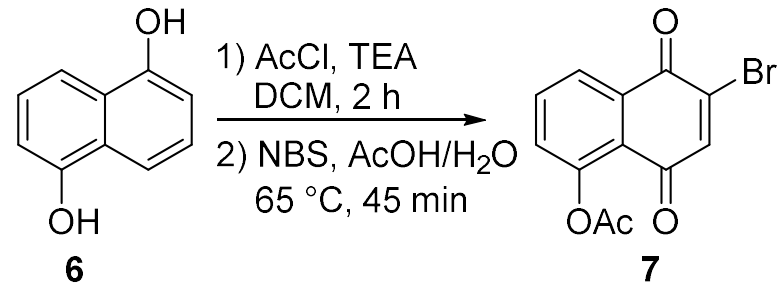

To a stirred solution of commercially available starting material 6 (4.8 g, $30 \mathrm{mmol})$ in $\mathrm{CH}_{2} \mathrm{Cl}_{2}(200 \mathrm{~mL})$ at $0{ }^{\circ} \mathrm{C}$ was added $\mathrm{AcCl}(5.3 \mathrm{~mL}, 75 \mathrm{mmol})$ and $\mathrm{Et}_{3} \mathrm{~N}(11 \mathrm{~mL}, 75$ mmol), the resulting mixture was stirred for $2 \mathrm{~h}$ at $25^{\circ} \mathrm{C}$ before saturated $\mathrm{NaHCO}_{3}$ was added. The aqueous phase was extracted with $\mathrm{CH}_{2} \mathrm{Cl}_{2}(50 \mathrm{~mL} \times 2)$. The combined organic phases were dried over anhydrous $\mathrm{Na}_{2} \mathrm{SO}_{4}$, filtered, and concentrated under vacuum. The residue was dissolved in acetic acid $(300 \mathrm{~mL})$ and was added over a period of $40 \mathrm{~min}$, with stirring, to a pre-heated solution of NBS (20.8 g, $120 \mathrm{mmol})$ dissolved in acetic acid $(300 \mathrm{~mL})$ and water $(600 \mathrm{~mL})$ at $65^{\circ} \mathrm{C}$. The reaction mixture was stirred at $65{ }^{\circ} \mathrm{C}$ for $45 \mathrm{~min}$ and then poured into water $(600 \mathrm{~mL})$. The aqueous suspension was extracted with $\mathrm{CH}_{2} \mathrm{Cl}_{2}(150 \mathrm{~mL} \times 4)$. The combined organic phases were washed with water $(500 \mathrm{~mL} \times 4)$ and dried over anhydrous $\mathrm{Na}_{2} \mathrm{SO}_{4}$, filtered, and the solvent was removed under vacuum to give yellow solid, recrystallization from ethanol afforded 7 (5.6 g, 63\% for two steps) as a yellow solid.

7: $R_{\mathrm{f}}=0.4$ (petroleum ether:EtOAc 10:1); ${ }^{1} \mathbf{H}$ NMR (400 MHz, $\left.\mathbf{C D C l}_{3}\right) \delta 8.14(\mathrm{dd}, J$ $=7.8,1.2 \mathrm{~Hz}, 1 \mathrm{H}), 7.77(\mathrm{t}, J=7.9 \mathrm{~Hz}, 1 \mathrm{H}), 7.42(\mathrm{dd}, J=8.0,1.2 \mathrm{~Hz}, 1 \mathrm{H}), 7.39(\mathrm{~s}, 1 \mathrm{H})$, $2.44(\mathrm{~s}, 3 \mathrm{H}) .{ }^{13} \mathbf{C}$ NMR (101 MHz, CDCl 3$) \delta 181.24,177.74,169.57,150.18,141.76$, 138.85, 135.26, 132.89, 130.66, 126.68, 123.46, 21.38. IR (neat): v 3052, 1774, 1660, $1592,1513,1265,1194,1094,773,751,689,662,634 \mathrm{~cm}^{-1}$. 


\section{Synthesis of $8^{2}$}<smiles>[2H]OC1=CC(=O)c2cc1ccc(=O)c2[B]</smiles>

1) but-3-enoic acid

$\mathrm{AgNO}_{3},\left(\mathrm{NH}_{4}\right)_{2} \mathrm{~S}_{2} \mathrm{O}_{8}$

$\mathrm{MeCN} / \mathrm{H}_{2} \mathrm{O}, 65^{\circ} \mathrm{C}, 2 \mathrm{~h}$

2) $3 \mathrm{~N} \mathrm{H}_{2} \mathrm{SO}_{4}$

$\mathrm{EtOH}$, reflux, $4 \mathrm{~h}$

3) $\mathrm{BnBr}, \mathrm{Ag}_{2} \mathrm{O}$

$\mathrm{DCM}, 24 \mathrm{~h}$

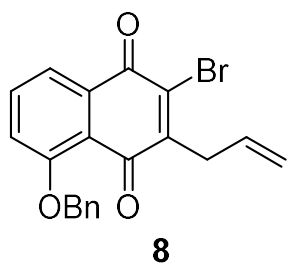

To a solution of $7(5.6 \mathrm{~g}, 18 \mathrm{mmol})$ in $\mathrm{MeCN}(200 \mathrm{~mL})$ at $25^{\circ} \mathrm{C}$, was added $\mathrm{AgNO}_{3}$ (1.6 $\mathrm{g}, 9 \mathrm{mmol})$ and vinylacetic acid $(2.4 \mathrm{~mL}, 28 \mathrm{mmol})$, the reaction mixture was heated to $65^{\circ} \mathrm{C}$, the system was then charged with $\mathrm{N}_{2}$. $\left(\mathrm{NH}_{4}\right)_{2} \mathrm{~S}_{2} \mathrm{O}_{8}(8.4 \mathrm{~g}, 37 \mathrm{mmol})$ in degassed $\mathrm{H}_{2} \mathrm{O}(60 \mathrm{~mL})$ was then added dropwise to the reaction mixture at $65{ }^{\circ} \mathrm{C}$ over a period of $30 \mathrm{~min}$. The reaction mixture was stirred at $65^{\circ} \mathrm{C}$ for $3 \mathrm{~h}$ and then cooled to $25{ }^{\circ} \mathrm{C}$. The reaction system was extracted with EtOAc $(150 \times 3 \mathrm{~mL})$, and the combined organic phases were washed with brine $(150 \mathrm{~mL})$, dried over anhydrous $\mathrm{Na}_{2} \mathrm{SO}_{4}$, filtered, and concentrated under vacuum to provide the crude allylation intermediate as a yellow solid.

A solution of the crude allylation intermediate and $3 \mathrm{~N} \mathrm{H}_{2} \mathrm{SO}_{4}(65 \mathrm{~mL})$ in ethanol $(300$ $\mathrm{mL}$ ) was heated to reflux and stirred for $4 \mathrm{~h}$ before it was cooled to $25^{\circ} \mathrm{C}$. Most of the solvent was removed under vacuum, the aqueous phase was then extracted with $\mathrm{CH}_{2} \mathrm{Cl}_{2}$ ( $100 \mathrm{~mL} \times 3)$, the combined organic phases were washed sequentially with $\mathrm{H}_{2} \mathrm{O}$ (200 $\mathrm{mL} \times 2$ ) and brine, dried over anhydrous $\mathrm{Na}_{2} \mathrm{SO}_{4}$, filtered, and concentrated under vacuum to provide the crude hydrolysis intermediate as a brown oil.

To a solution of the crude hydrolysis intermediate in $\mathrm{CH}_{2} \mathrm{Cl}_{2}(200 \mathrm{~mL})$ at $25{ }^{\circ} \mathrm{C}$ was added $\mathrm{BnBr}(5.5 \mathrm{~mL}, 46 \mathrm{mmol})$ and $\mathrm{Ag}_{2} \mathrm{O}(5.7 \mathrm{~g}, 25 \mathrm{mmol})$. The reaction mixture was stirred at $25{ }^{\circ} \mathrm{C}$ for $18 \mathrm{~h}$ and then filtered through a pad of celite, the filtrate was concentrated to offer crude product. Flash column chromatography (silica gel, petroleum ether:EtOAc 10:1) provided 8 (3.3 g, 48\% for three steps) as a yellow solid. 8: $R_{\mathrm{f}}=0.45$ (petroleum ether:EtOAc 10:1); ${ }^{1} \mathbf{H}$ NMR (400 MHz, $\left.\mathbf{C D C l}_{3}\right) \delta 7.85-7.79$ (m, 1H), $7.60(\mathrm{t}, J=8.1 \mathrm{~Hz}, 1 \mathrm{H}), 7.55(\mathrm{~d}, J=7.5 \mathrm{~Hz}, 2 \mathrm{H}), 7.42(\mathrm{t}, J=7.5 \mathrm{~Hz}, 2 \mathrm{H}), 7.33$ (t, $J=7.3 \mathrm{~Hz}, 2 \mathrm{H}), 5.88(\mathrm{ddt}, J=16.7,10.0,6.6 \mathrm{~Hz}, 1 \mathrm{H}), 5.31(\mathrm{~s}, 2 \mathrm{H}), 5.27$ (dd, $J=$ 16.8, $1.7 \mathrm{~Hz}, 3 \mathrm{H}), 5.14(\mathrm{dd}, J=10.1,1.6 \mathrm{~Hz}, 1 \mathrm{H}), 3.63(\mathrm{~d}, J=6.5 \mathrm{~Hz}, 3 \mathrm{H}) .{ }^{13} \mathbf{C} \mathbf{N M R}$ 
(101 MHz, CDCl $) \delta 180.37,178.50,159.25,151.03,136.99,136.24,135.05,133.69$, $132.12,129.10(2 \mathrm{C}), 128.41,127.05$ (2C), 120.93, 120.29, $120.11,118.47,71.37,36.07$. HRMS (ESI): calcd for $\mathrm{C}_{20} \mathrm{H}_{15} \mathrm{BrO}_{3}[\mathrm{M}+\mathrm{Na}]^{+}: 405.0097$, found: 405.0103. IR (neat): v $3063,3035,2913,1661,1602,1582,1449,1381,1257,1219,1149,974,831,771$, $733,695 \mathrm{~cm}^{-1}$.

\section{Synthesis of 9}<smiles>C=CCC1=C(Br)C(=O)c2cccc(OCc3ccccc3)c2C1=O</smiles>

8

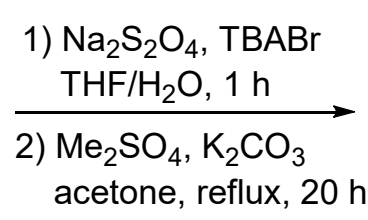

acetone, reflux, $20 \mathrm{~h}$<smiles>C=CCc1c(Br)c(OC)c2cccc(OCc3ccccc3)c2c1OC</smiles>

9

To a solution of $8(3.3 \mathrm{~g}, 8.6 \mathrm{mmol})$ in THF $(100 \mathrm{~mL})$ and $\mathrm{H}_{2} \mathrm{O}(100 \mathrm{~mL})$ was added $\mathrm{Na}_{2} \mathrm{~S}_{2} \mathrm{O}_{4}(17 \mathrm{~g}, 98 \mathrm{mmol})$ and $\mathrm{TBABr}(14 \mathrm{mg})$ at $25{ }^{\circ} \mathrm{C}$, the reaction mixture was charged with $\mathrm{N}_{2}$ and stirred vigorously for $1 \mathrm{~h}$. The reaction mixture was extracted with EtOAc $(100 \mathrm{~mL} \times 3)$, the combined organic layers were washed with brine $(100 \mathrm{~mL})$, dried over $\mathrm{Na}_{2} \mathrm{SO}_{4}$, and concentrated. The resulting gray solid was dissolved in acetone (200 mL), $\mathrm{Me}_{2} \mathrm{SO}_{4}(5 \mathrm{~mL}, 53 \mathrm{mmol})$ and $\mathrm{K}_{2} \mathrm{CO}_{3}(6.4 \mathrm{~g}, 46 \mathrm{mmol})$ were then added to the reaction mixture, the reaction mixture was heated to reflux, and kept at reflux for $20 \mathrm{~h}$. The solvent was removed under vacuum, the residue was then treated with water, and the biphasic reaction mixture was extracted with EtOAc $(50 \mathrm{~mL} \times 3)$, the combined organic layers were dried over $\mathrm{Na}_{2} \mathrm{SO}_{4}$ and concentrated. The residue was purified by flash column chromatography (silica gel, petroleum ether:EtOAc 50:1) to give 9 (2.9 g, $82 \%$ for two steps) as a colorless oil.

9: $R_{\mathrm{f}}=0.35$ (petroleum ether:EtOAc $\left.=50: 1\right) ;{ }^{1} \mathbf{H}$ NMR $\left(400 \mathbf{M H z}, \mathbf{C D C l}_{3}\right) \delta 7.79(\mathrm{~d}$, $J=8.4 \mathrm{~Hz}, 1 \mathrm{H}), 7.61(\mathrm{~d}, J=7.6 \mathrm{~Hz}, 2 \mathrm{H}), 7.52-7.36(\mathrm{~m}, 4 \mathrm{H}), 7.04(\mathrm{~d}, J=7.7 \mathrm{~Hz}, 1 \mathrm{H})$, 6.12 (ddt, $J=16.4,10.9,5.8 \mathrm{~Hz}, 1 \mathrm{H}), 5.27$ (s, 2H), 5.13 (d, $J=10.2 \mathrm{~Hz}, 1 \mathrm{H}), 5.08$ (d, $J=16.8 \mathrm{~Hz}, 1 \mathrm{H}), 4.00(\mathrm{~s}, 3 \mathrm{H}), 3.85(\mathrm{~d}, J=5.1 \mathrm{~Hz}, 2 \mathrm{H}), 3.74(\mathrm{~s}, 3 \mathrm{H}) .{ }^{13} \mathbf{C}$ NMR (101 MHz, CDCl3) $\delta 155.27,151.41,149.69,136.87,136.31,130.52,129.82,128.58(2 \mathrm{C})$, $127.97,127.67$ (2C), 126.65, 120.48, 117.59, 115.74, 115.46, 108.74, 71.60, 63.23, 
61.20, 34.35. HRMS (ESI): calcd for $\mathrm{C}_{22} \mathrm{H}_{21} \mathrm{BrO}_{3}[\mathrm{M}+\mathrm{H}]^{+}: 413.0747$, found: 413.0748 .

IR (neat): v 3068, 2997, 2837, 1611, 1560, 1497, 1449, 1361, 1322, 1263, 1165, 1050, $976,909,746,698 \mathrm{~cm}^{-1}$. 


\section{Synthesis of $4^{2}$}<smiles>C=CCc1c(Br)c(OC)c2cccc(OCc3ccccc3)c2c1OC</smiles>

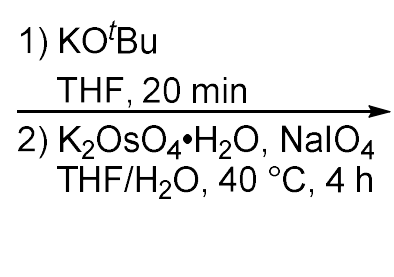<smiles>COc1c(Br)c(C=O)c(OC)c2c(OC)cccc12</smiles>

To a solution of $9(2.6 \mathrm{~g}, 6.3 \mathrm{mmol})$ in THF $(150 \mathrm{~mL})$ was added $t$-BuOK $(3 \mathrm{~g}, 12 \mathrm{mmol})$ and the reaction mixture was stirred for $20 \mathrm{~min}$ at $25^{\circ} \mathrm{C}$. The reaction mixture was then quenched with $\mathrm{H}_{2} \mathrm{O}(50 \mathrm{~mL})$ and the biphasic reaction mixture was extracted with EtOAc $(100 \mathrm{~mL} \times 2)$. The combined organic layers were dried and concentrated to afford the crude double bond migrated intermediate.

To a solution of the crude double bond migrated intermediate in THF $(80 \mathrm{~mL})$ and $\mathrm{H}_{2} \mathrm{O}$ $(40 \mathrm{~mL})$ at $25{ }^{\circ} \mathrm{C}$ was added $\mathrm{K}_{2} \mathrm{OsO}_{4}(116 \mathrm{mg}, 0.31 \mathrm{mmol})$ and $\mathrm{NaIO}_{4}(4 \mathrm{~g}, 19 \mathrm{mmol})$. The reaction mixture was heated to $40{ }^{\circ} \mathrm{C}$ and kept stirring for $4 \mathrm{~h}$. The reaction mixture was then quenched with sat. aqueous $\mathrm{Na}_{2} \mathrm{~S}_{2} \mathrm{O}_{3}(40 \mathrm{~mL})$ and stirred vigorously for 30 min. The biphasic reaction mixture was extracted with EtOAc $(100 \mathrm{~mL} \times 3)$ and then the combined organic layers were washed with brine $(100 \mathrm{~mL})$, dried over $\mathrm{Na}_{2} \mathrm{SO}_{4}$, and concentrated. The crude product was purified by flash column chromatography (silica gel, petroleum ether:EtOAc 10:1) to provided 4 (1.9 g, 75\% for two steps) as a colorless oil.

4: $R_{\mathrm{f}}=0.45$ (petroleum ether:EtOAc $\left.=10: 1\right) ;{ }^{1} \mathbf{H}$ NMR (400 MHz, CDCl $) \delta 10.49(\mathrm{~s}$, 1H), $7.76(\mathrm{~d}, J=8.4 \mathrm{~Hz}, 1 \mathrm{H}), 7.61-7.51(\mathrm{~m}, 3 \mathrm{H}), 7.43(\mathrm{t}, J=7.3 \mathrm{~Hz}, 2 \mathrm{H}), 7.40-7.34$ (m, 1H), 7.07 (d, $J=7.8 \mathrm{~Hz}, 1 \mathrm{H}), 5.24$ (s, 2H), 3.96 (s, 3H), 3.78 (s, 3H). ${ }^{13} \mathbf{C}$ NMR $\left(\mathbf{1 0 1} \mathbf{~ M H z}, \mathbf{C D C l}_{3}\right) \delta 190.80,158.99,156.83,150.47,136.19,133.94,130.39,128.71$ (2C), 128.28, 127.72 (2C), 125.08, 120.09, 115.43, 111.37, 109.08, 71.50, 65.40, 61.31. HRMS (ESI): calcd for $\mathrm{C}_{20} \mathrm{H}_{17} \mathrm{BrO}_{4}[\mathrm{M}+\mathrm{Na}]^{+}: 423.0202$, found: 423.0206 . IR (neat): v 2937, 2842, 1693, 1607, 1549, 1448, 1364, 1265, 1126, 1048, 971, 930, 813, 735, $698 \mathrm{~cm}^{-1}$. 


\section{Synthesis of 11}<smiles>CC(=O)c1ccc(I)c(O)c1</smiles>

10<smiles>Cc1ccc(I)c(OBr)c1</smiles>

11

To a stirred solution of the $10(2.8 \mathrm{~g}, 10 \mathrm{mmol})$ in DMF $(40 \mathrm{~mL})$ at $25{ }^{\circ} \mathrm{C}$ was added $\mathrm{K}_{2} \mathrm{CO}_{3}(2 \mathrm{~g}, 15 \mathrm{mmol})$ and $\mathrm{BnBr}(1.8 \mathrm{~mL}, 15 \mathrm{mmol})$. The resulting mixture was stirred for $1 \mathrm{~h}$ before $\mathrm{H}_{2} \mathrm{O}(150 \mathrm{~mL})$ was added. The reaction system was extracted with EtOAc $(100 \mathrm{~mL} \times 3)$. The combined organic layers were washed with $\mathrm{H}_{2} \mathrm{O}(200 \mathrm{~mL} \times 3)$ and brine $(200 \mathrm{~mL})$, the organic phase was dried $\left(\mathrm{Na}_{2} \mathrm{SO}_{4}\right)$ and concentrated in vacuo. The residue was purified by flash column chromatography (silica gel, petroleum ether:EtOAc $=20: 1)$ to afford $11(3.4 \mathrm{~g}, 92 \%)$ as a white amorphous solid.

11: $R_{\mathrm{f}}=0.5$ (petroleum ether:EtOAc $\left.=10: 1\right) ;{ }^{1} \mathbf{H} \mathbf{N M R}\left(400 \mathbf{M H z}, \mathbf{C D C l}_{3}\right) \delta 7.93(\mathrm{~d}$, $J=8.1 \mathrm{~Hz}, 1 \mathrm{H}), 7.61-7.52(\mathrm{~m}, 3 \mathrm{H}), 7.51-7.34(\mathrm{~m}, 4 \mathrm{H}), 5.25(\mathrm{~s}, 2 \mathrm{H}), 3.96(\mathrm{~s}, 3 \mathrm{H})$. ${ }^{13}$ C NMR (101 MHz, CDCl3) $\delta 166.56,157.31,139.62,136.03,131.54,128.63$ (2C), 128.07, 127.14 (2C), 123.60, 112.73, 93.50, 70.99, 52.42. HRMS (ESI): calcd for $\mathrm{C}_{15} \mathrm{H}_{13} \mathrm{IO}_{3}[\mathrm{M}+\mathrm{Na}]^{+}:$390.9801, found: 390.9817. IR (neat): v 3031, 2948, 2878, 1719 , $1574,1435,1406,1379,1288,1233,1107,1016,903,875,832,793,755,696,626$ $\mathrm{cm}^{-1}$. 


\section{Synthesis of 5}

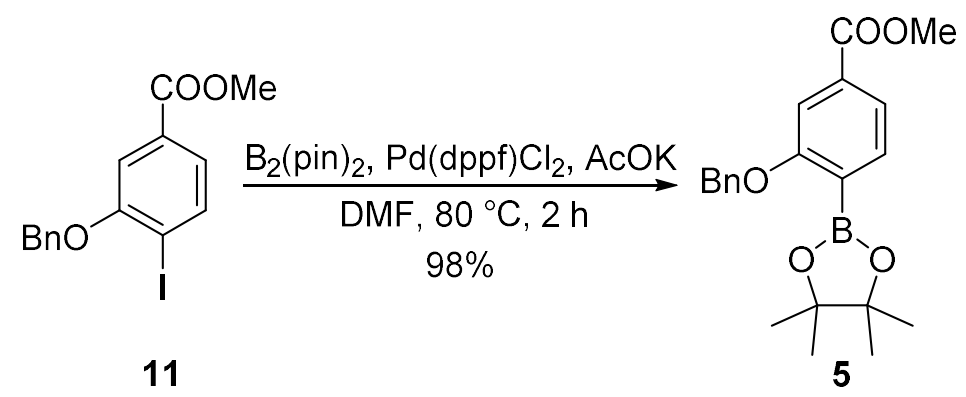

To a solution of $11(3 \mathrm{~g}, 8 \mathrm{mmol})$ in DMF $(30 \mathrm{~mL})$ at $25^{\circ} \mathrm{C}$ was added $\mathrm{B}_{2}(\text { pin })_{2}(2.5 \mathrm{~g}$, $10 \mathrm{mmol})$, $\mathrm{AcOK}(1.6 \mathrm{~g}, 16 \mathrm{mmol})$, and $\mathrm{Pd}(\mathrm{dppf}) \mathrm{Cl}_{2}(900 \mathrm{mg}, 1.2 \mathrm{mmol})$, the reaction system was charged with $\mathrm{N}_{2}$. The reaction mixture was heated to $80{ }^{\circ} \mathrm{C}$ and stirred for $2 \mathrm{~h}$ at $80{ }^{\circ} \mathrm{C}$ followed by cooling. The reaction mixture was then quenched with $\mathrm{H}_{2} \mathrm{O}$ $(150 \mathrm{~mL})$ and the reaction mixture was extracted with EtOAc $(100 \mathrm{~mL} \times 3)$, the combined organic layers were washed with $\mathrm{H}_{2} \mathrm{O}(200 \mathrm{~mL} \times 3)$ and brine $(200 \mathrm{~mL})$, dried $\left(\mathrm{Na}_{2} \mathrm{SO}_{4}\right)$, and concentrated in vacuo. The residue was purified by flash column chromatography (silica gel, petroleum ether:EtOAc $=10: 1)$ to provide $5(2.9 \mathrm{~g}, 98 \%)$ as a white amorphous solid.

5: $R_{\mathrm{f}}=0.3$ (petroleum ether:EtOAc $\left.=20: 1\right) ;{ }^{1} \mathbf{H}$ NMR (400 MHz, $\left.\mathbf{C D C l}_{3}\right) \delta 7.74(\mathrm{~d}, J$ $=7.5 \mathrm{~Hz}, 1 \mathrm{H}), 7.67-7.57(\mathrm{~m}, 4 \mathrm{H}), 7.38(\mathrm{t}, J=7.5 \mathrm{~Hz}, 2 \mathrm{H}), 7.30(\mathrm{t}, J=7.3 \mathrm{~Hz}, 1 \mathrm{H})$, 5.18 (s, 2H), 3.93 (s, 3H), 1.37 (s, 12H). ${ }^{13} \mathbf{C}$ NMR (101 MHz, CDCl 3$) \delta 167.01,163.04$, $137.18,136.44,133.66,128.20$ (3C), 127.47, 126.81 (2C), 121.67, 112.22, 83.89, 83.52, 69.97, 52.27, 25.04 (2C), 24.94 (2C). IR (neat): v 2983, 1724, 1610, 1564, 1502, 1412, $1377,1349,1283,1232,1171,1123,1065,1029,962,907,852,808,747,697,662 \mathrm{~cm}^{-}$ 1. 


\section{Synthesis of 3}

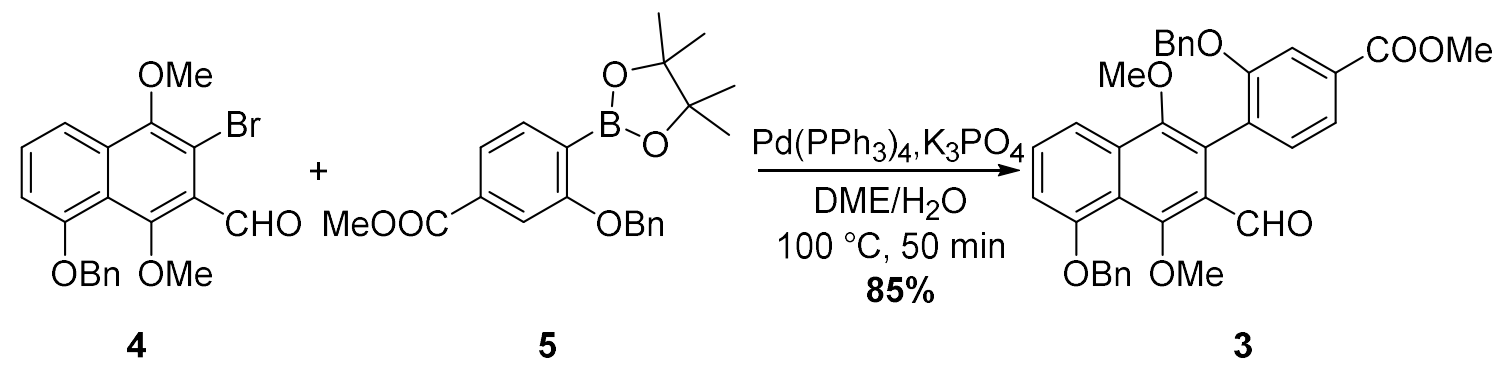

To a sealed tube was added substrate $4(1 \mathrm{~g}, 2.5 \mathrm{mmol}), 5(1.4 \mathrm{~g}, 3.8 \mathrm{mmol}), \mathrm{K}_{3} \mathrm{PO}_{4}$ (1.6 g, $7.5 \mathrm{mmol}), \mathrm{Pd}\left(\mathrm{PPh}_{3}\right)_{4}(288 \mathrm{mg}, 1.25 \mathrm{mmol}), \mathrm{DME}(36 \mathrm{~mL})$ and $\mathrm{H}_{2} \mathrm{O}(12 \mathrm{~mL})$, the reaction system was charged with $\mathrm{N}_{2}$, and then stirred at $100{ }^{\circ} \mathrm{C}$ for $50 \mathrm{~min}$. After being cooled to $25^{\circ} \mathrm{C}$, the reaction mixture was extracted with EtOAc $(20 \mathrm{~mL} \times 3)$, the combined organic layers were dried $\left(\mathrm{Na}_{2} \mathrm{SO}_{4}\right)$, and concentrated in vacuo. The residue was purified by flash column chromatography ( silica gel, petroleum ether:EtOAc $=6: 1$ ) to provide 3 (1.2 g, 85\%) as a yellow amorphous solid.

3: $R_{\mathrm{f}}=0.31$ (petroleum ether:EtOAc $\left.=4: 1\right)$; ${ }^{1} \mathbf{H}$ NMR (400 MHz, $\left.\mathbf{C D C l}_{3}\right) \delta 10.37(\mathrm{~s}$, 1H), $7.84-7.78(\mathrm{~m}, 2 \mathrm{H}), 7.72(\mathrm{~s}, 1 \mathrm{H}), 7.57$ (t, $J=8.1 \mathrm{~Hz}, 3 \mathrm{H}), 7.47-7.36(\mathrm{~m}, 4 \mathrm{H})$, $7.25-7.16(\mathrm{~m}, 5 \mathrm{H}), 7.08$ (d, $J=7.8 \mathrm{~Hz}, 1 \mathrm{H}), 5.28(\mathrm{~s}, 2 \mathrm{H}), 5.14-5.00(\mathrm{~m}, 2 \mathrm{H}), 3.96$ (s, 3H), 3.74 (s, 3H), 3.43 (s, 3H). ${ }^{13}$ C NMR (101 MHz, CDCl3) $\delta 191.38,167.38$, 159.02, 156.93, 156.14, 150.41, 136.99, 136.77, 134.86, 131.54, 131.00, 131.00, 129.86, 129.00 (2C), 128.66 (2C), 128.50, 128.03, 127.98 (2C), 127.46 (2C), 126.28, $126.15,122.72,120.88,116.15,112.80,109.33,71.81,70.59,65.44,61.51,52.56$. HRMS (ESI): calcd for $\mathrm{C}_{35} \mathrm{H}_{30} \mathrm{O}_{7}[\mathrm{M}+\mathrm{Na}]^{+}: 585.1884$, found: 585.1890. IR (neat): $\mathrm{v}$ 2996, 2839, 1718, 1686, 1609, 1566, 1500, 1439, 1415, 1363, 1334, 1285, 1229, 1117 , $1052,876,736,697 \mathrm{~cm}^{-1}$. 


\section{Synthesis of 2}

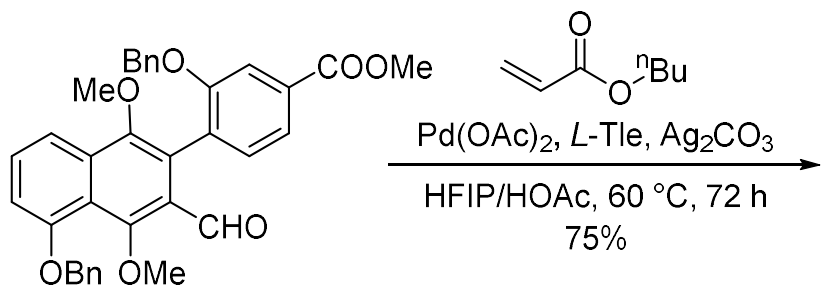

3

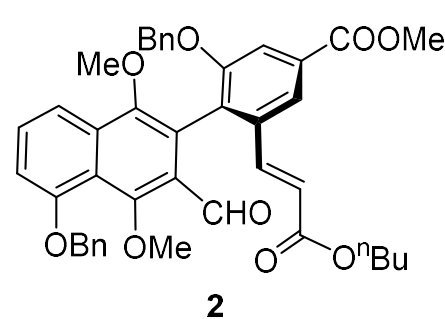

2

To an oven-dried Schlenk tube was added substrate 3 (1.2 g, $2.1 \mathrm{mmol})$, butyl acrylate (1.2 mL, $8.4 \mathrm{mmol}), \mathrm{Pd}(\mathrm{OAc})_{2}(47 \mathrm{mg}, 0.21 \mathrm{mmol}), L$-Tle $(112 \mathrm{mg}, 0.84 \mathrm{mmol})$, $\mathrm{Ag}_{2} \mathrm{CO}_{3}(2.4 \mathrm{~g}, 8.4 \mathrm{mmol}), \mathrm{HFIP}(16 \mathrm{~mL})$ and HOAc $(4 \mathrm{~mL})$. The tube was then charged with $\mathrm{N}_{2}$, the mixture was stirred for $72 \mathrm{~h}$ at $60^{\circ} \mathrm{C}$ followed by cooling. The resulting mixture was diluted with DCM and filtered through a pad of celite and concentrated in vacuo. The residue was purified by column chromatography (silica gel, petroleum ether:EtOAc $=6: 1)$ to provide $2(1.08 \mathrm{~g}, 75 \%, 99 \%$ ee $)$ as a yellow amorphous solid. 2: $R_{\mathrm{f}}=0.29$ (petroleum ether:EtOAc $\left.=4: 1\right) ;[\alpha]_{\mathrm{D}}{ }^{23}+59.7\left(\right.$ c 1.00, $\left.\mathrm{CHCl}_{3}\right) ;{ }^{1} \mathbf{H} \mathbf{~ N M R}$ (400 MHz, CDCl $) \delta 10.41(\mathrm{~s}, 1 \mathrm{H}), 8.15(\mathrm{~s}, 1 \mathrm{H}), 7.78(\mathrm{~d}, J=8.4 \mathrm{~Hz}, 1 \mathrm{H}), 7.71(\mathrm{~s}, 1 \mathrm{H})$, $7.60(\mathrm{t}, J=5.1 \mathrm{~Hz}, 3 \mathrm{H}), 7.46(\mathrm{t}, J=7.5 \mathrm{~Hz}, 2 \mathrm{H}), 7.40(\mathrm{~d}, J=7.3 \mathrm{~Hz}, 1 \mathrm{H}), 7.35(\mathrm{~d}, J=$ $16.0 \mathrm{~Hz}, 1 \mathrm{H}), 7.18$ (t, $J=3.3 \mathrm{~Hz}, 3 \mathrm{H}), 7.11$ (d, $J=7.0 \mathrm{~Hz}, 3 \mathrm{H}), 6.49$ (d, $J=16.0 \mathrm{~Hz}$, 1H), $5.30(\mathrm{~s}, 2 \mathrm{H}), 5.04(\mathrm{~d}, J=13.0 \mathrm{~Hz}, 2 \mathrm{H}), 4.11-4.00(\mathrm{~m}, 2 \mathrm{H}), 3.98(\mathrm{~s}, 3 \mathrm{H}), 3.78(\mathrm{~s}$, $3 \mathrm{H}), 3.43(\mathrm{~s}, 3 \mathrm{H}), 1.53(\mathrm{p}, J=6.6 \mathrm{~Hz}, 2 \mathrm{H}), 1.25(\mathrm{~h}, J=6.2,5.5 \mathrm{~Hz}, 3 \mathrm{H}), 0.82(\mathrm{t}, J=7.3$

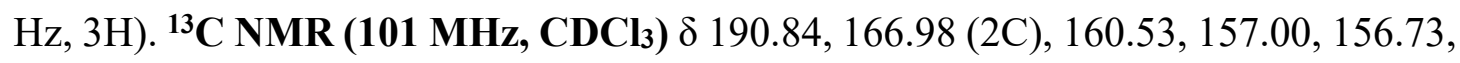
$150.41,142.13,136.85,136.75,134.96,134.80,132.55,130.82,130.20,129.00$ (2C), 128.62 (2C), 128.50, 127.96 (3C), 127.14 (2C), 125.83, 123.36, 121.22, 120.69, 120.55, 116.33, 113.44, 109.64, 71.89, 70.79, 65.75, 64.53, 61.42, 52.68, 30.88, 19.40, 13.93. HRMS (ESI): calcd for $\mathrm{C}_{42} \mathrm{H}_{40} \mathrm{O}_{9}[\mathrm{M}+\mathrm{Na}]^{+}:$711.2564, found: 711.2578. IR (neat): $v$ 2956, 2872, 1715, 1683, 1642, 1608, 1567, 1492, 1442, 1362, 1334, 1271, 1229, 1175 , 1053, 1011, 973, 938, 768, 735, $697 \mathrm{~cm}^{-1}$. HPLC separation (Chiralpak ${ }^{\circledR}$ AD-H, $n$ hexane $/ i$-PrOH 70:30, $1.5 \mathrm{~mL} / \mathrm{min}$, detection at $254 \mathrm{~nm}$ ): $\operatorname{tr}$ (major) $=27.3 \mathrm{~min}$, $t r$ $($ minor $)=14.1 \mathrm{~min}, 99 \%$ ee. 


\section{HPLC spectra of $\mathbf{2}$ :}

$\mathrm{mV}$

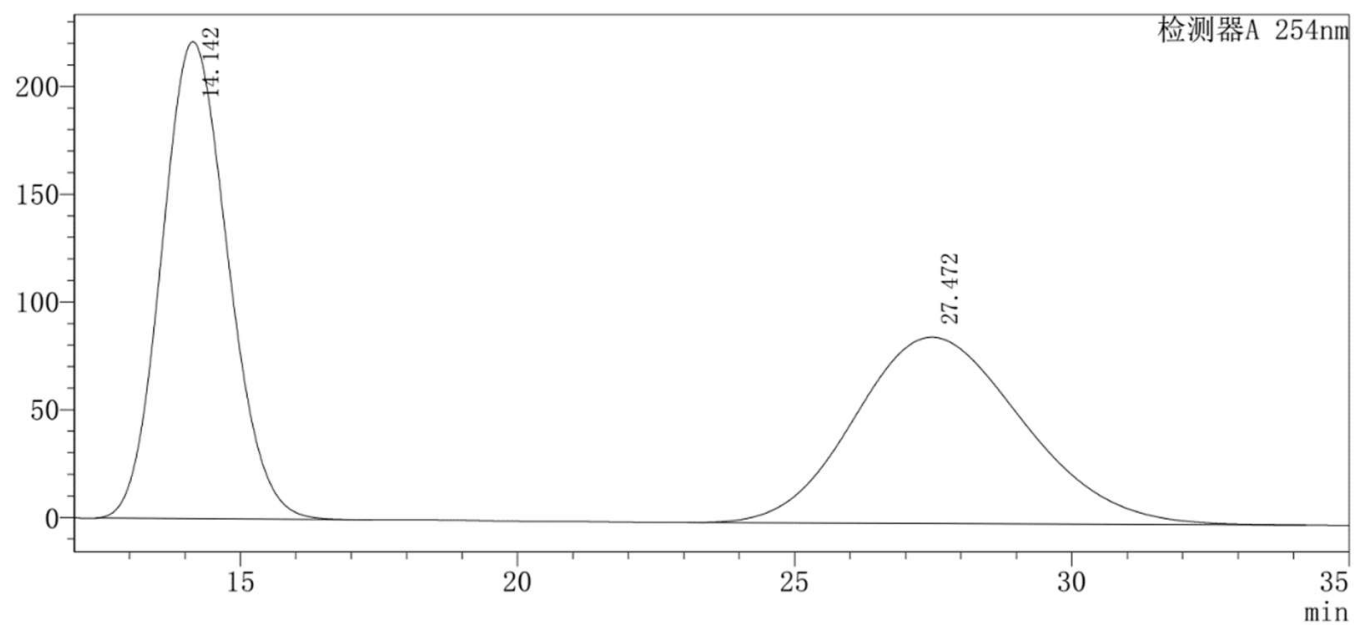

〈峰表〉

检测器A $254 \mathrm{~nm}$

\begin{tabular}{|c|c|c|c|c|c|}
\hline 峰号 & 保留时间 & 面积 & 高度 & 浓度 & 面积\% \\
\hline 1 & 14.142 & 18422658 & 221430 & 50.082 & 50.082 \\
\hline 2 & 27.472 & 18362430 & 86524 & 49.918 & 49.918 \\
\hline 总计 & & 36785087 & 307954 & & 100.000 \\
\hline
\end{tabular}

$\mathrm{mV}$

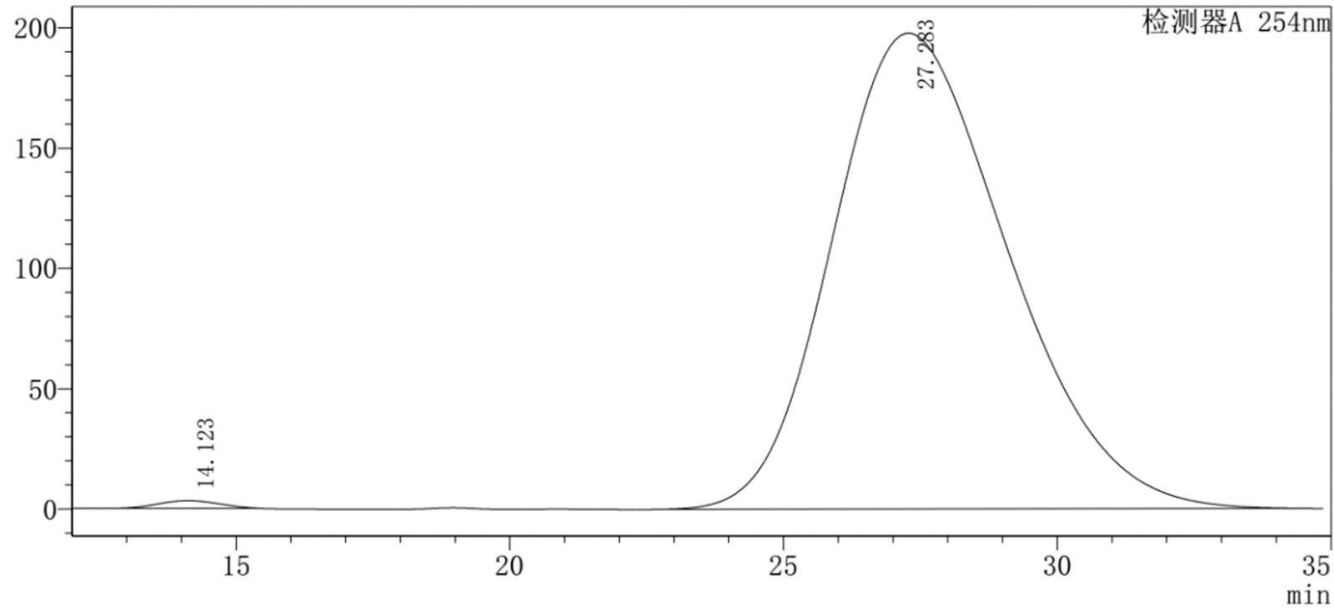

〈峰表〉

检测器A $254 \mathrm{~nm}$

\begin{tabular}{|c|c|c|c|c|c|}
\hline 峰号 & 保留时间 & 面积 & 高度 & 浓度 & 面积\% \\
\hline 1 & 14.123 & 250151 & 3210 & 0.564 & 0.564 \\
\hline 2 & 27.283 & 44109234 & 197791 & 99.436 & 99.436 \\
\hline 总计 & & 44359385 & 201000 & & 100.000 \\
\hline
\end{tabular}




\section{Synthesis of 1}
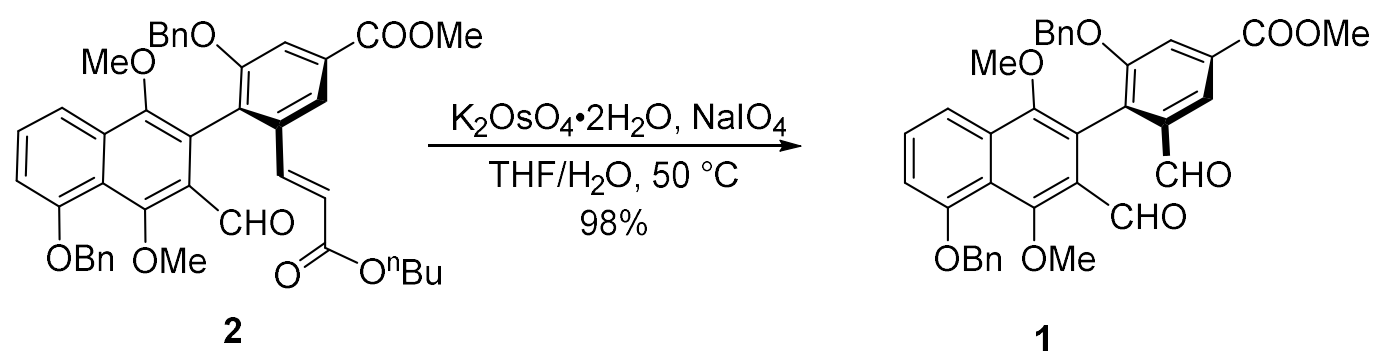

To a solution of $2(1.2 \mathrm{~g}, 1.7 \mathrm{mmol})$ in THF $(90 \mathrm{~mL})$ and $\mathrm{H}_{2} \mathrm{O}(45 \mathrm{~mL})$ was added $\mathrm{K}_{2} \mathrm{OsO}_{4}(60 \mathrm{mg}, 0.16 \mathrm{mmol})$ and $\mathrm{NaIO}_{4}(4 \mathrm{~g}, 17 \mathrm{mmol})$ at $25^{\circ} \mathrm{C}$, the reaction mixture was stirred at $50{ }^{\circ} \mathrm{C}$ for $12 \mathrm{~h}$. Another portion of $\mathrm{K}_{2} \mathrm{OsO}_{4}(60 \mathrm{mg}, 0.16 \mathrm{mmol})$ and $\mathrm{NaIO}_{4}$ ( $4 \mathrm{~g}, 17 \mathrm{mmol}$ ) was added to the reaction system, and the mixture was stirred at $50{ }^{\circ} \mathrm{C}$ for an additional $12 \mathrm{~h}$, then another portion of $\mathrm{NaIO}_{4}(4 \mathrm{~g}, 17 \mathrm{mmol})$ was added, and the mixture was stirred at $50{ }^{\circ} \mathrm{C}$ for additional $4 \mathrm{~h}$, The reaction mixture was then quenched with sat. aqueous $\mathrm{Na}_{2} \mathrm{~S}_{2} \mathrm{O}_{3}(100 \mathrm{~mL})$ and stirred vigorously for $30 \mathrm{~min}$. The biphasic reaction mixture was extracted with EtOAc $(100 \mathrm{~mL} \times 3)$ and then the combined organic layers were washed with brine $(100 \mathrm{~mL})$, dried $\left(\mathrm{Na}_{2} \mathrm{SO}_{4}\right)$, and concentrated. The crude product was purified by flash column chromatography (silica gel, petroleum ether:EtOAc $=4: 1)$ to provided $\mathbf{1}(1.03 \mathrm{~g}, 98 \%, 98 \% e e)$ as a yellow oil. $\mathbf{1}: R_{\mathrm{f}}=0.27$ (petroleum ether:EtOAc $=4: 1) .[\alpha]_{\mathrm{D}}{ }^{23}+41.9\left(\mathrm{c} 1.00, \mathrm{CHCl}_{3}\right) .{ }^{1} \mathbf{H}$ NMR (400 MHz, $\left.\mathbf{C D C l}_{3}\right) \delta 10.44(\mathrm{~s}, 1 \mathrm{H}), 9.75(\mathrm{~s}, 1 \mathrm{H}), 8.39$ (s, 1H), 7.91 (s, 1H), 7.77 (d, $J=8.4 \mathrm{~Hz}$, 1H), $7.63-7.55(\mathrm{~m}, 3 \mathrm{H}), 7.45(\mathrm{t}, J=7.4 \mathrm{~Hz}, 2 \mathrm{H}), 7.39$ (t, $J=7.3 \mathrm{~Hz}, 1 \mathrm{H}), 7.24-7.17$ (m, 3H), $7.17-7.09(\mathrm{~m}, 3 \mathrm{H}), 5.29(\mathrm{~s}, 2 \mathrm{H}), 5.13-4.98(\mathrm{~m}, 2 \mathrm{H}), 3.97(\mathrm{~s}, 3 \mathrm{H}), 3.74(\mathrm{~s}$, 3H), 3.35 (s, 3H). ${ }^{13}$ C NMR (101 MHz, CDCl $) \delta 191.41,190.83,166.62,160.50$, $156.96,156.67,150.61,136.62,136.46,135.26,134.58,134.47,131.17,130.43$, 129.01 (2C), 128.72 (2C), 128.55, 128.21, 127.97 (2C), 127.38 (2C), 126.11, 121.82, $121.25,121.11,116.93,116.25,109.88,71.88,71.10,65.71,61.11,52.75$. HRMS (ESI): calcd for $\mathrm{C}_{36} \mathrm{H}_{30} \mathrm{O}_{8}[\mathrm{M}+\mathrm{Na}]^{+}:$613.1833, found: 613.1848. IR (neat): v 3004, $2847,1722,1687,1607,1569,1506,1447,1364,1331,1291,1221,1053,971,909$, 745, $700 \mathrm{~cm}^{-1}$. HPLC separation (Chiralpak ${ }^{\circledR}$ AD-H, $n$-hexane/i-PrOH 70:30, 1.5 $\mathrm{mL} / \mathrm{min}$, detection at $254 \mathrm{~nm}): \operatorname{tr}$ (major) $=13.5 \mathrm{~min}, \operatorname{tr}$ (minor) $=22.8 \mathrm{~min}, 98 \%$ ee. 


\section{HPLC spectra of $\mathbf{1}$}

$\mathrm{mV}$

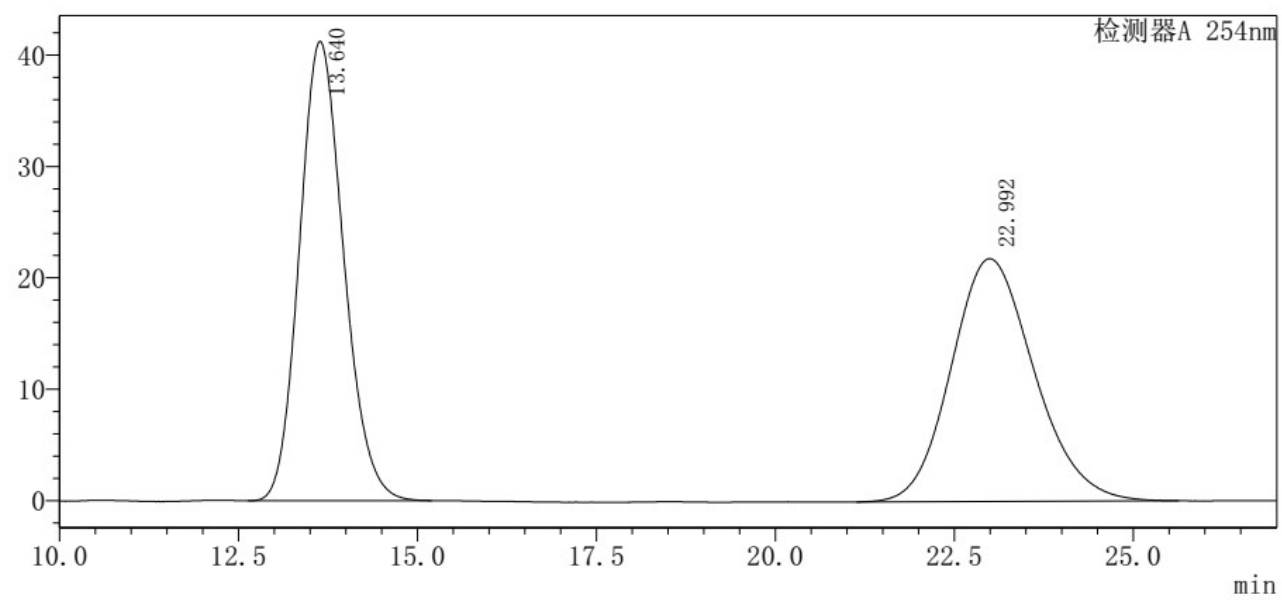

〈峰表〉

检测器A $254 \mathrm{~nm}$

\begin{tabular}{|c|c|c|c|c|} 
检测器A $254 \mathrm{~nm}$ \\
\hline 峰号 & 保留时间 & 面积 & 高度 & 面积\% \\
\hline 1 & 13.640 & 1772774 & 41239 & 50.191 \\
\hline 2 & 22.992 & 1759269 & 21805 & 49.809 \\
\hline 总计 & & 3532043 & 63044 & 100.000 \\
\hline
\end{tabular}

$\mathrm{mV}$

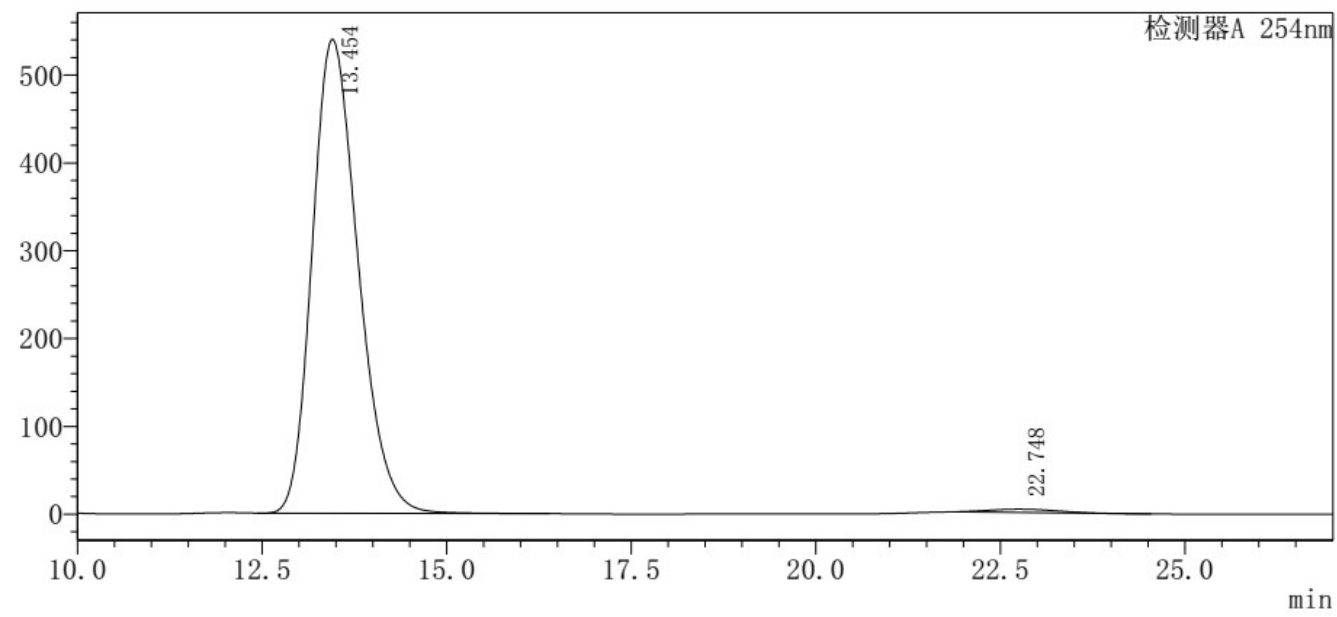

〈峰表〉

检测器A $254 \mathrm{~nm}$

\begin{tabular}{|c|c|c|c|c|c|}
\hline 峰号 & 保留时间 & 面积 & 高度 & 浓度 & 面积\% \\
\hline 1 & 13.454 & 23332620 & 539722 & 98.984 & 98.984 \\
\hline 2 & 22.748 & 239429 & 3736 & 1.016 & 1.016 \\
\hline 总计 & & 23572049 & 543459 & & 100.000 \\
\hline
\end{tabular}




\section{Synthesis of 12}<smiles>COc1cc(C(C)=O)cc(C=O)c1-c1c(CO)c(COc2ccccc2)c(OC)c2c(OC)cccc12</smiles>

1
1) $\mathrm{Sml}_{2}, \mathrm{THF}$

2) $m-\mathrm{Me}-\mathrm{BzCl}$

$0{ }^{\circ} \mathrm{C}$ $54 \%$

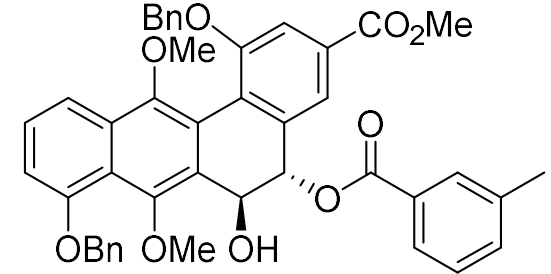

12

To a solution of dialdehyde 1 (100 $\mathrm{mg}, 0.17 \mathrm{mmol})$ in THF $(4.0 \mathrm{~mL})$ was added $\mathrm{SmI}_{2}$ $(0.1 \mathrm{M}, 3.5 \mathrm{~mL}, 0.35 \mathrm{mmol})$ at $0{ }^{\circ} \mathrm{C}$. After stirring for $10 \mathrm{~min}$, a solution of 3 methylbenzoyl chloride, $(34 \mu \mathrm{L}, 0.25 \mathrm{mmol})$ in THF $(0.5 \mathrm{~mL})$ was added and the reaction mixture was stirred overnight at $0{ }^{\circ} \mathrm{C}$. The reaction was quenched with $1 \mathrm{M}$ $\mathrm{HCl}$ at $0{ }^{\circ} \mathrm{C}$. The mixture was extracted with EtOAc $(10 \mathrm{~mL} \times 3)$ and the combined organic extracts were washed with brine $(20 \mathrm{~mL})$, dried $\left(\mathrm{Na}_{2} \mathrm{SO}_{4}\right)$ and concentrated in vacuo. The residue was purified by column chromatography (silica gel, petroleum ether:EtOAc $=3: 1)$ to afford $12(64 \mathrm{mg}, 54 \%, 98 \% e e)$ as a colorless amorphous. 12: $R_{\mathrm{f}}=0.6$ (petroleum ether:EtOAc $\left.=1: 1\right) ;[\alpha]_{\mathrm{D}}{ }^{23}+284.1\left(\mathrm{c} 0.43, \mathrm{CHCl}_{3}\right) ;{ }^{1} \mathbf{H} \mathbf{~ N M R}$ (400 MHz, CDCl $) \delta 8.07-7.97(\mathrm{~m}, 2 \mathrm{H}), 7.87(\mathrm{~s}, 1 \mathrm{H}), 7.57(\mathrm{~d}, J=7.4 \mathrm{~Hz}, 2 \mathrm{H}), 7.49$ (t, $J=8.1 \mathrm{~Hz}, 1 \mathrm{H}), 7.45-7.38(\mathrm{~m}, 5 \mathrm{H}), 7.36(\mathrm{~d}, J=7.3 \mathrm{~Hz}, 1 \mathrm{H}), 7.31$ (d, $J=9.3 \mathrm{~Hz}$, 1H), $7.25-7.15$ (m, 4H), 7.07 (d, $J=7.8 \mathrm{~Hz}, 2 \mathrm{H}), 6.26$ (d, $J=3.4 \mathrm{~Hz}, 1 \mathrm{H}), 5.58$ (s, 1H), $5.33-5.22(\mathrm{~m}, 3 \mathrm{H}), 5.13(\mathrm{~d}, J=11.8 \mathrm{~Hz}, 1 \mathrm{H}), 3.95(\mathrm{~s}, 3 \mathrm{H}), 3.75(\mathrm{~s}, 3 \mathrm{H}), 3.50$ (s, 3H), 2.14 (s, 3H), $1.71(\mathrm{~d}, J=6.6 \mathrm{~Hz}, 1 \mathrm{H}) .{ }^{13} \mathbf{C}$ NMR (101 MHz, CDCl3) $\delta 166.54$, $165.52,156.02,155.56,152.13,151.20,137.97,136.83,136.67,133.76,133.61$, $132.64,130.72,130.12,129.60,128.58(2 \mathrm{C}), 128.22(2 \mathrm{C}), 128.15,128.00,127.69(2 \mathrm{C})$, $127.66,127.33$ (2C), 126.91, 126.39, 126.11, 125.28, 120.95, 116.18, 116.02, 109.25, 73.09, 71.53, 71.28, 64.14, 63.58, 61.64, 52.36, 21.06. HRMS (ESI): calcd for $\mathrm{C}_{44} \mathrm{H}_{38} \mathrm{O}_{9}[\mathrm{M}+\mathrm{Na}]^{+}:$733.2408, found: 733.2420. IR (neat): v 3481, 2946, 2838, 1717, $1576,1497,1437,1364,1314,1267,1237,1196,1104,1061,977,895,739,698 \mathrm{~cm}^{-1}$. HPLC separation (Chiralpak® IB, $n$-hexane/i-PrOH 70:30, $1.0 \mathrm{~mL} / \mathrm{min}$, detection at $254 \mathrm{~nm}): \operatorname{tr}($ major $)=13.5 \mathrm{~min}, \operatorname{tr}($ minor $)=18.3 \mathrm{~min}, 98 \%$ ee. 


\section{HPLC spectra of 12:}

$\mathrm{mV}$

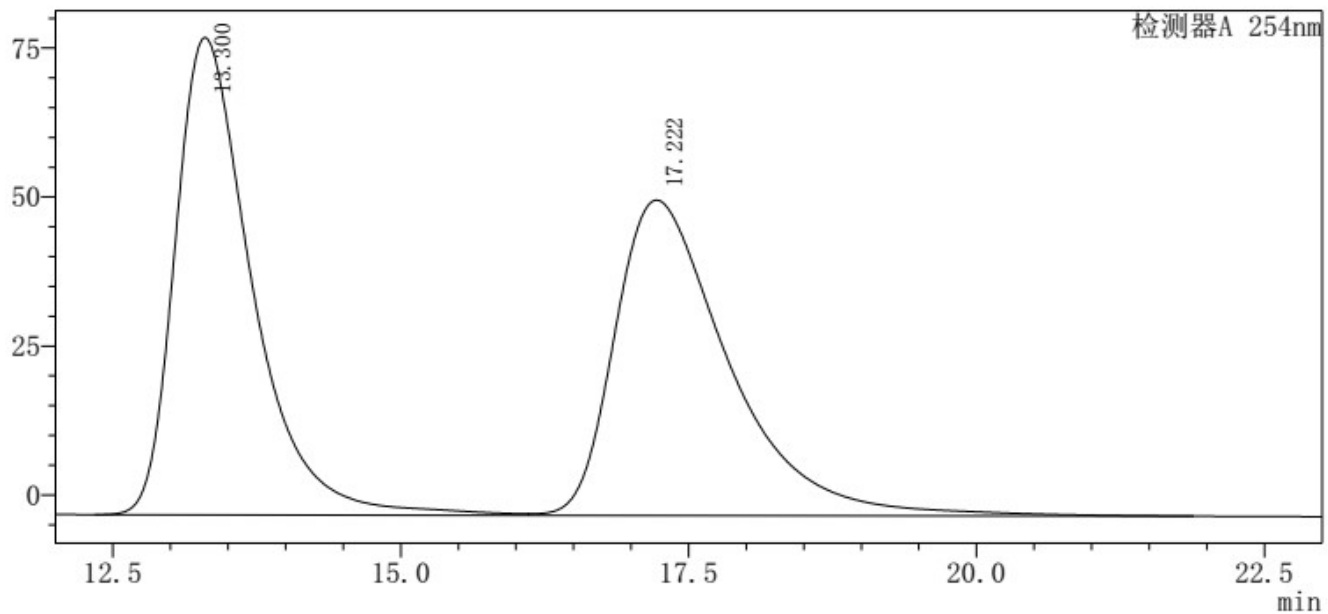

〈峰表〉

检测器A $254 \mathrm{~nm}$
\begin{tabular}{|r|r|c|c|c|c|}
\hline 峰号 & 保留时间 & 面积 & 高度 & 浓度 & 面积\% \\
\hline 1 & 13.300 & 3729672 & 80097 & 50.494 & 50.494 \\
\hline 2 & 17.222 & 3656706 & 52914 & 49.506 & 49.506 \\
\hline 总计 & & 7386378 & 133012 & & 100.000 \\
\hline
\end{tabular}

$\mathrm{mV}$

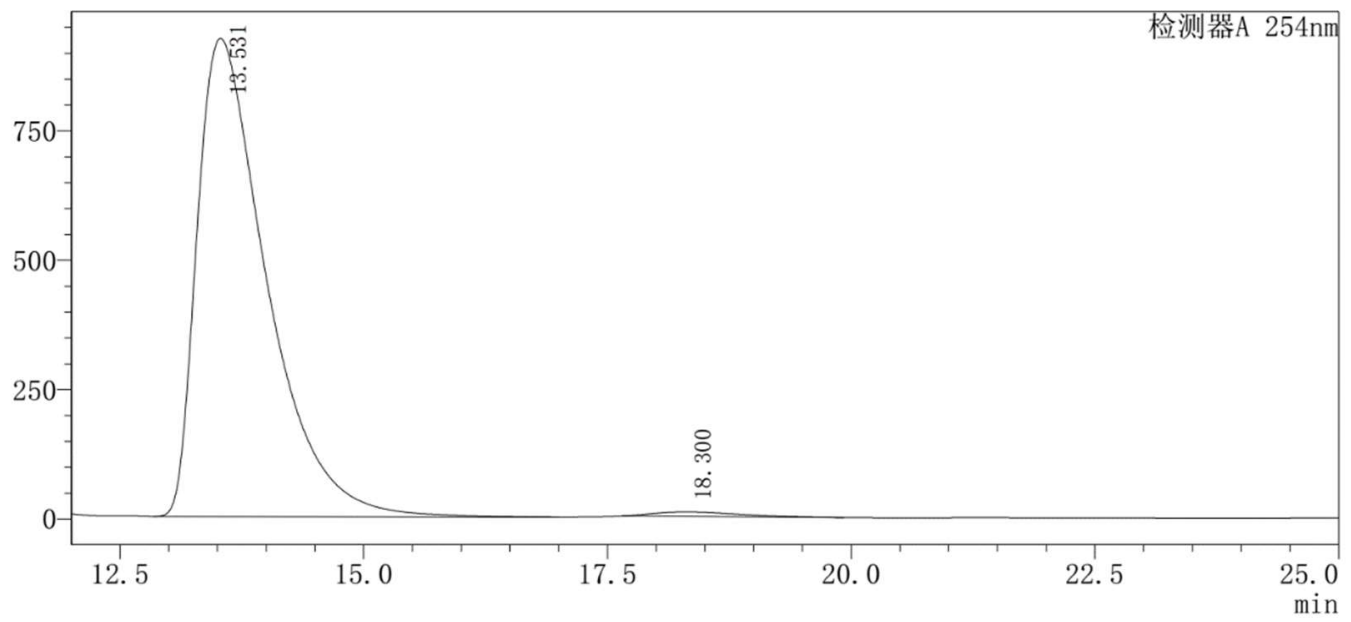

〈峰表〉

检测器A $254 \mathrm{~nm}$

\begin{tabular}{|r|r|c|c|c|c|}
\hline \multicolumn{1}{|c|}{ 峰号 } & 保留时间 & 面积 & 高度 & 浓度 & 面积\% \\
\hline 1 & 13.531 & 45816388 & 924013 & 98.843 & 98.843 \\
\hline 2 & 18.300 & 536250 & 8618 & 1.157 & 1.157 \\
\hline 总计 & & 46352638 & 932632 & & 100.000 \\
\hline
\end{tabular}




\section{Synthesis of TAN-1085}

The final steps of the synthesis were identical to literature report ${ }^{3-4}$.

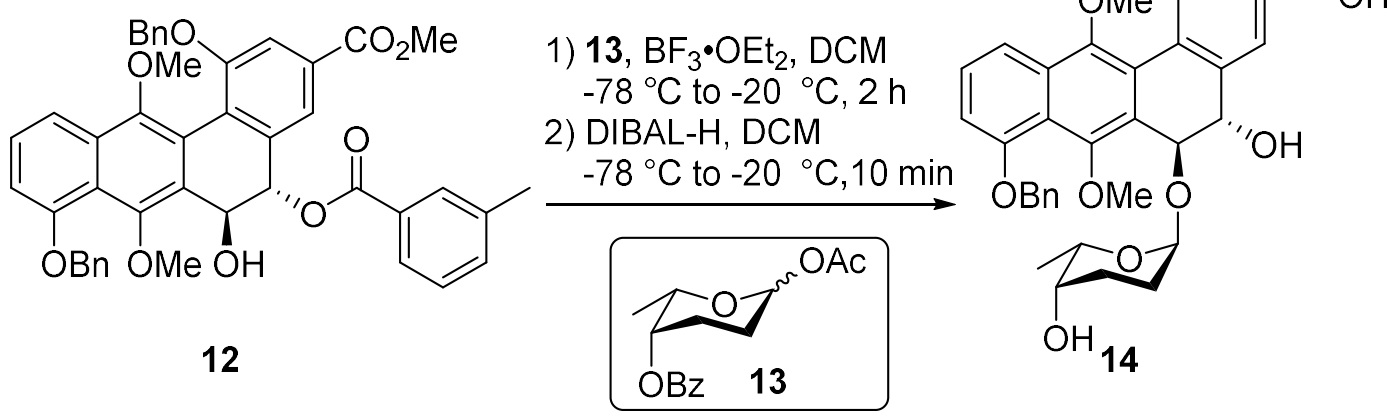

To a solution of 12 (40 mg, $0.057 \mathrm{mmol})$ and $13(40 \mathrm{mg}, 0.14 \mathrm{mmol})$ in $\mathrm{CH}_{2} \mathrm{Cl}_{2}(5.0$ $\mathrm{mL})$ was added a solution of $\mathrm{BF}_{3} \cdot \mathrm{OEt}_{2}(7.7 \mathrm{uL}, 0.063 \mathrm{mmol})$ in $\mathrm{CH}_{2} \mathrm{Cl}_{2}(0.1 \mathrm{~mL})$ at $78{ }^{\circ} \mathrm{C}$. After stirring for $2 \mathrm{~h}$ at $-20^{\circ} \mathrm{C}$, the reaction was quenched with saturated aqueous $\mathrm{NaHCO}_{3}$ and $\mathrm{Et}_{3} \mathrm{~N}$. The crude products were extracted with $\mathrm{CH}_{2} \mathrm{Cl}_{2}(10 \mathrm{~mL} \times 3)$, and the combined organic extracts were washed with brine $(15 \mathrm{~mL})$, dried $\left(\mathrm{Na}_{2} \mathrm{SO}_{4}\right)$, and concentrated in vacuo to afford crude glycosylated product.

To a solution of the crude glycoside in $\mathrm{CH}_{2} \mathrm{Cl}_{2}(4 \mathrm{~mL})$ was added DIBAL-H $(1.00 \mathrm{M}$ in $n$ hexane, $0.5 \mathrm{~mL}, 0.5 \mathrm{mmol})$ at $-78^{\circ} \mathrm{C}$. The reaction system was warmed up to -20 ${ }^{\circ} \mathrm{C}$ and stirred for $10 \mathrm{~min}$ at $-20{ }^{\circ} \mathrm{C}$. The reaction was quenched by adding $25 \%$ aqueous potassium sodium tartrate followed by stirring for $2 \mathrm{~h}$ at room temperature. The mixture was extracted with $\mathrm{CH}_{2} \mathrm{Cl}_{2}(10 \mathrm{~mL} \times 3)$, and the combined organic organic phases were washed with brine $(15 \mathrm{~mL})$, dried $\left(\mathrm{Na}_{2} \mathrm{SO}_{4}\right)$, and concentrated in vacuo. The residue was purified by flash column chromatography (silica gel, $\mathrm{CH}_{2} \mathrm{Cl}_{2}: \mathrm{MeOH}=20: 1$ ) to afford 14 (35 mg, 90\%) as a white amorphous.

14: $R_{\mathrm{f}}=0.45\left(\mathrm{CH}_{2} \mathrm{Cl}_{2}: \mathrm{MeOH}=10: 1\right) ;[\alpha]_{\mathrm{D}}{ }^{23}+199.4\left(\mathrm{c} 0.50, \mathrm{CHCl}_{3}\right) ;{ }^{1} \mathbf{H}$ NMR (400 MHz, CDCl $) \delta 7.97(\mathrm{~d}, J=8.4 \mathrm{~Hz}, 1 \mathrm{H}), 7.58(\mathrm{~d}, J=7.5 \mathrm{~Hz}, 2 \mathrm{H}), 7.44(\mathrm{~d}, J=7.7 \mathrm{~Hz}$, $3 \mathrm{H}), 7.40(\mathrm{t}, J=7.0 \mathrm{~Hz}, 2 \mathrm{H}), 7.34(\mathrm{t}, J=7.2 \mathrm{~Hz}, 1 \mathrm{H}), 7.26-7.17(\mathrm{~m}, 3 \mathrm{H}), 7.11(\mathrm{~s}, 1 \mathrm{H})$, $7.06(\mathrm{~s}, 1 \mathrm{H}), 7.01(\mathrm{~d}, J=7.7 \mathrm{~Hz}, 1 \mathrm{H}), 5.47(\mathrm{~d}, J=3.4 \mathrm{~Hz}, 1 \mathrm{H}), 5.28(\mathrm{~d}, J=11.8 \mathrm{~Hz}$, 1H), $5.24-5.14(\mathrm{~m}, 2 \mathrm{H}), 5.12$ (d, $J=3.3 \mathrm{~Hz}, 1 \mathrm{H}), 5.08$ (d, J=11.9 Hz, 1H), 4.93 (d, $J=3.4 \mathrm{~Hz}, 1 \mathrm{H}), 4.72(\mathrm{~s}, 2 \mathrm{H}), 3.94(\mathrm{q}, J=6.5 \mathrm{~Hz}, 1 \mathrm{H}), 3.79$ (s, 3H), $3.53(\mathrm{~s}, 3 \mathrm{H}), 3.31$ (s, 1H), $2.04-1.68(\mathrm{~m}, 4 \mathrm{H}), 1.66-1.55(\mathrm{~m}, 1 \mathrm{H}), 1.51-1.38(\mathrm{~m}, 1 \mathrm{H}), 1.20-1.09(\mathrm{~m}$, 
1H), $0.91(\mathrm{~d}, J=6.6 \mathrm{~Hz}, 3 \mathrm{H}) .{ }^{13} \mathbf{C}$ NMR (101 MHz, $\left.\mathbf{C D C l}_{3}\right) \delta 156.52,155.20,151.69$, $150.91,141.95,138.65,137.41,137.30,132.85,128.44$ (2C), 128.19 (2C), 127.74, 127.43, 127.30 (2C), 127.13 (2C), 126.59, 124.57, 121.11, 120.35, 120.29, 119.59, 116.33, 113.02, 109.60, 95.10, 71.69, 70.78, 69.77, 68.60, 67.49, 66.64, 64.93, 63.32, 61.13, 25.51, 23.61, 16.91. HRMS (ESI): calcd for $\mathrm{C}_{41} \mathrm{H}_{42} \mathrm{O}_{9}[\mathrm{M}+\mathrm{Na}]^{+}$: 701.2721, found: 701.2722 .
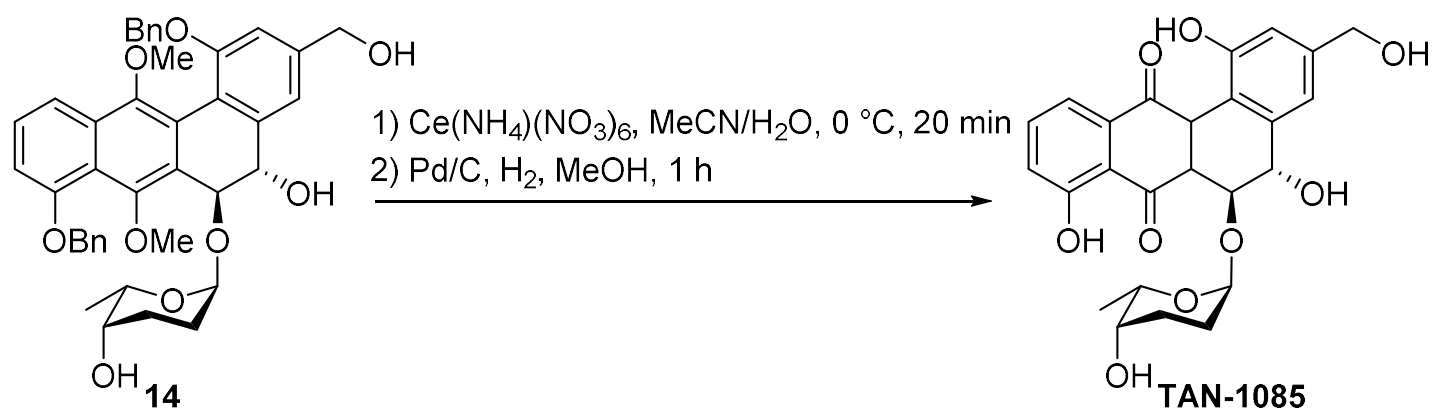

To a solution of $14(23 \mathrm{mg}, 0.034 \mathrm{mmol})$ in $\mathrm{CH}_{3} \mathrm{CN}(3 \mathrm{~mL})$ was added a solution of $\mathrm{Ce}\left(\mathrm{NH}_{4}\right)_{2}\left(\mathrm{NO}_{3}\right)_{6}(74 \mathrm{mg}, 0.14 \mathrm{mmol})$ in $\mathrm{H}_{2} \mathrm{O}(1 \mathrm{~mL})$ at $0{ }^{\circ} \mathrm{C}$. After stirring for $20 \mathrm{~min}$ at $0{ }^{\circ} \mathrm{C}$, the reaction was quenched by adding $\mathrm{H}_{2} \mathrm{O}(10 \mathrm{~mL})$. The reaction mixture was extracted with EtOAc $(10 \mathrm{~mL} \times 3)$, and the combined organic phases were washed with saturated aqueous $\mathrm{NaHCO}_{3}(10 \mathrm{~mL})$ and brine $(10 \mathrm{~mL})$, the mixture was then dried $\left(\mathrm{Na}_{2} \mathrm{SO}_{4}\right)$, and concentrated in vacuo to provide crude intermediate.

To a solution of this crude intermediate in $\mathrm{MeOH}(3.0 \mathrm{~mL})$ was added $10 \% \mathrm{Pd} / \mathrm{C}(2.0$ $\mathrm{mg}$ ), the system was charged with $\mathrm{H}_{2}$. After stirring at room temperature for $1 \mathrm{~h}$ under $\mathrm{H}_{2}$, the reaction mixture was filtered through a pad of celite, the filtrate was concentrated in vacuo. The residue was purified by PTLC $\left(\mathrm{CH}_{2} \mathrm{Cl}_{2}: \mathrm{MeOH}=10: 1\right)$ to afford TAN-1085 (8.0 mg, 50\%) as a reddish brown solid.

TAN-1085: $R_{\mathrm{f}}=0.25(\mathrm{DCM}: \mathrm{MeOH}=10: 1) ;[\alpha]_{\mathrm{D}}{ }^{23}+70($ c $0.02, \mathrm{MeOH}) ;{ }^{1} \mathbf{H}$ NMR (400 MHz, МeOH) $\delta 7.68(\mathrm{t}, J=7.9 \mathrm{~Hz}, 1 \mathrm{H}), 7.58(\mathrm{dd}, J=7.5,1.1 \mathrm{~Hz}, 1 \mathrm{H}), 7.26$ (dd, $J=8.4,1.0 \mathrm{~Hz}, 1 \mathrm{H}), 6.96(\mathrm{~d}, J=1.5 \mathrm{~Hz}, 1 \mathrm{H}), 6.92(\mathrm{~d}, J=1.5 \mathrm{~Hz}, 1 \mathrm{H}), 5.19$ (d, $J=2.9$ $\mathrm{Hz}, 1 \mathrm{H}), 5.16(\mathrm{brd}, J=3.5 \mathrm{~Hz}, 1 \mathrm{H}), 4.71(\mathrm{~d}, J=2.9 \mathrm{~Hz}, 1 \mathrm{H}), 4.61(\mathrm{~s}, 2 \mathrm{H}), 3.53-3.42$ (m, 1H), 3.21 (brs, $1 \mathrm{H}), 1.95-1.82(\mathrm{~m}, 1 \mathrm{H}), 1.58-1.47(\mathrm{~m}, 1 \mathrm{H}), 1.47-1.38(\mathrm{~m}, 1 \mathrm{H})$, $1.35-1.28(\mathrm{~m}, 1 \mathrm{H}), 0.73(\mathrm{~d}, J=6.5 \mathrm{~Hz}, 3 \mathrm{H}) .{ }^{13} \mathbf{C}$ NMR (101 MHz, MeOH) $\delta 190.05$, $186.03,162.13,157.65,147.74,144.21,141.30,141.00,137.23,135.70,124.42$, 
$120.82,119.83,116.89,116.15,116.00,97.75,70.37,69.38,68.34,67.76,64.51,26.39$, $24.60,16.92$. 


\section{References}

(1) Grunwell, J. R.; Karipides, A.; Wigal, C. T.; Heinzman, S. W.; Parlow, J.; Surso, J. A.; Clayton, L.; Fleitz, F. J.; Daffner, M.; Stevens, J. E., The Formal Oxidative Addition of Electron-Rich Transoid Dienes to Bromonaphthoquinones. J. Org. Chem. 1991, 56, 91-95.

(2) Nicolaou, K. C.; Li, H.; Nold, A. L.; Pappo, D.; Lenzen, A., Total Synthesis of Kinamycins C, F, and J. J. Am. Chem. Soc. 2007, 129, 10356-10357.

(3) Ohmori, K.; Mori, K.; Ishikawa, Y.; Tsuruta, H.; Kuwahara, S.; Harada, N.; Suzuki, K., Concise Total Synthesis and Structure Assignment of TAN-1085. Angew. Chem., Int. Ed. 2004, 43, 3167-3171.

(4) Mori, K.; Ohmori, K.; Suzuki, K., Stereochemical Relay via Axially Chiral Styrenes: Asymmetric Synthesis of the Antibiotic TAN-1085. Angew. Chem., Int. Ed. 2009, $48,5633-5637$. 


\section{Spectra}

7: ${ }^{1} \mathrm{H}$ NMR, $400 \mathrm{MHz}, \mathrm{CDCl}_{3}$

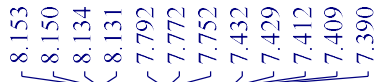

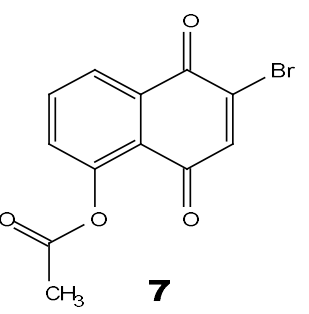

$\mathrm{CDCl}_{3}$

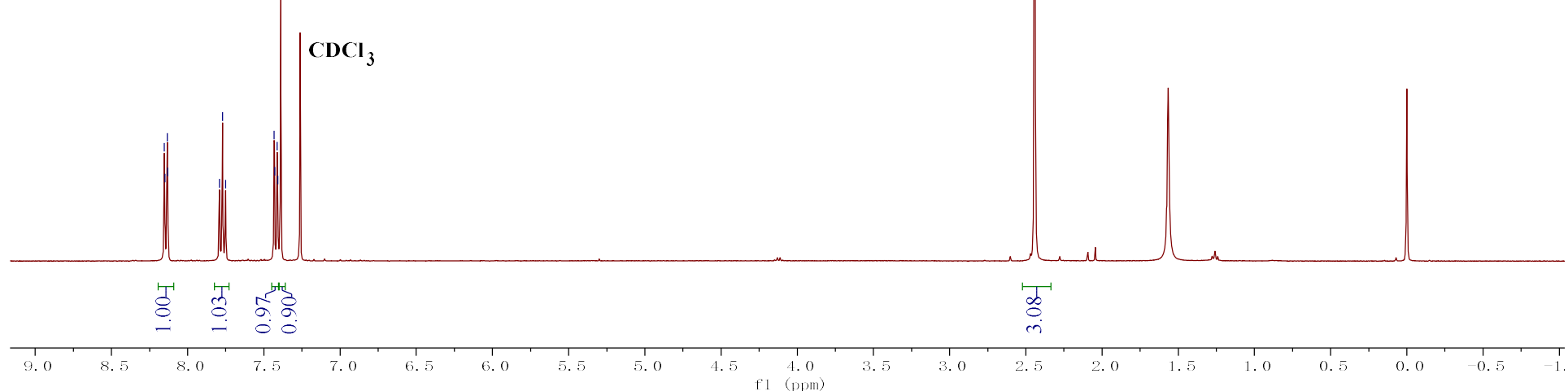


7: ${ }^{13} \mathrm{C} \mathrm{NMR}, 400 \mathrm{MHz}, \mathrm{CDCl}_{3}$
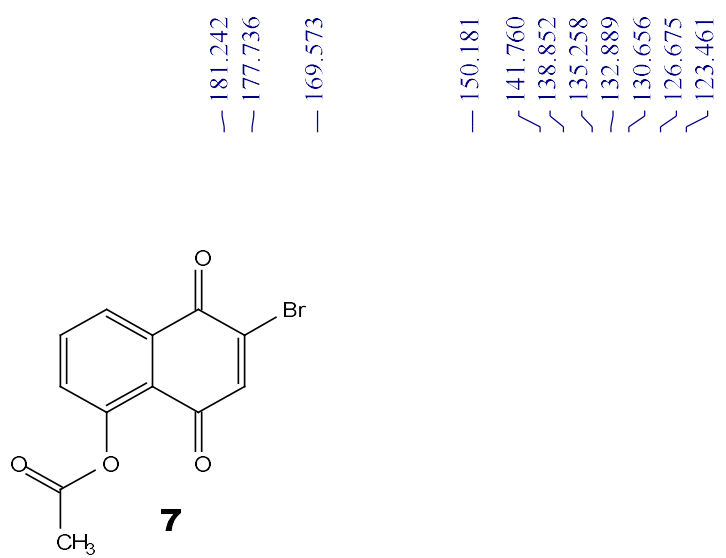

$\mathrm{CDCl}_{3}$

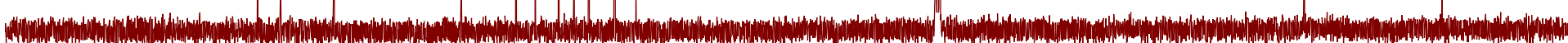

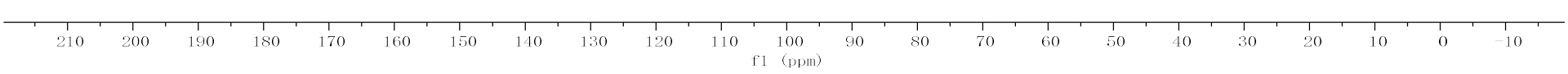


8: ${ }^{1} \mathrm{H} \mathrm{NMR,} 400 \mathrm{MHz}, \mathrm{CDCl}_{3}$

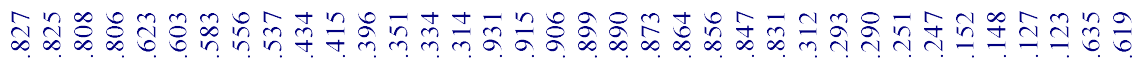

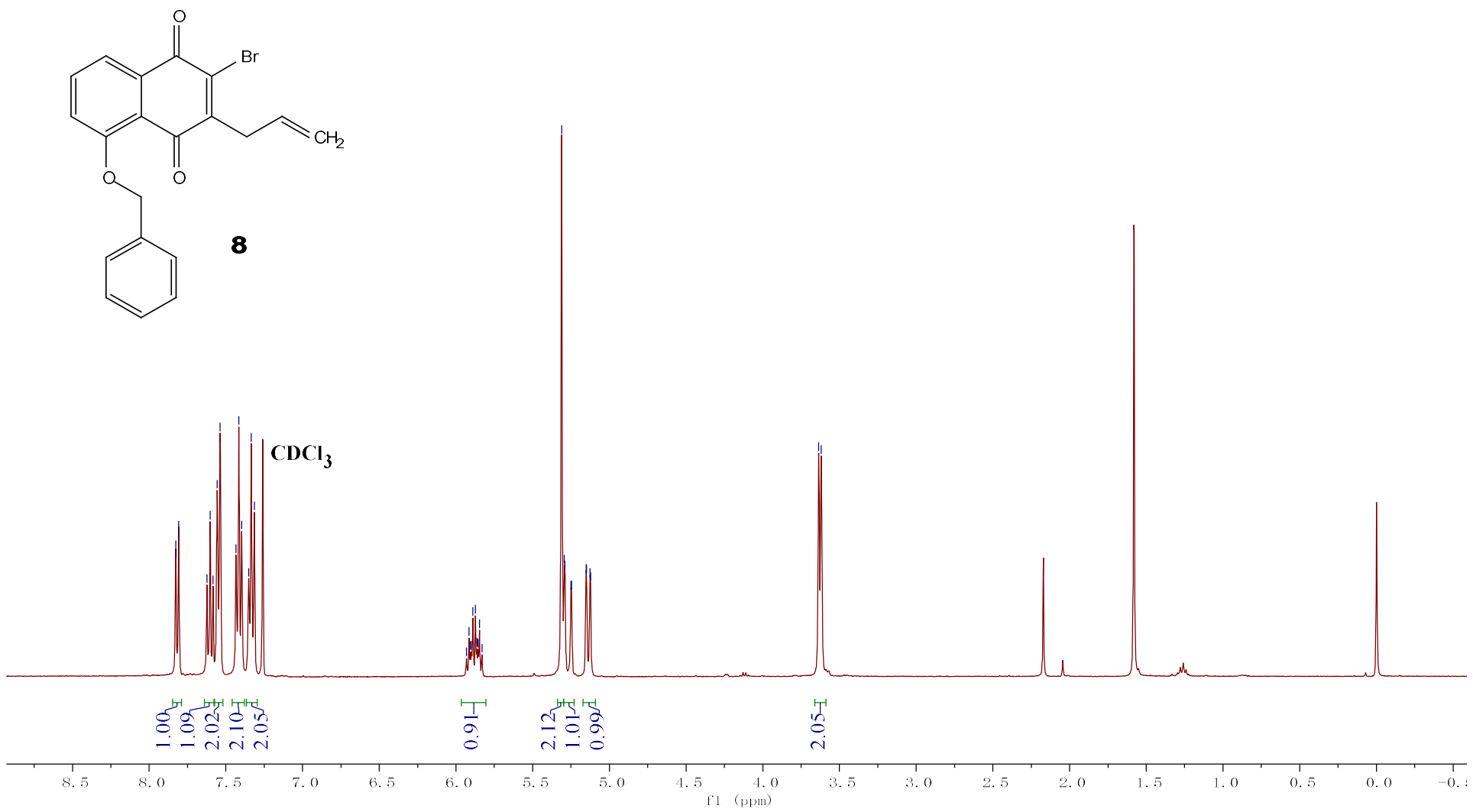


8: ${ }^{13} \mathrm{C} \mathrm{NMR}, 400 \mathrm{MHz}, \mathrm{CDCl}_{3}$

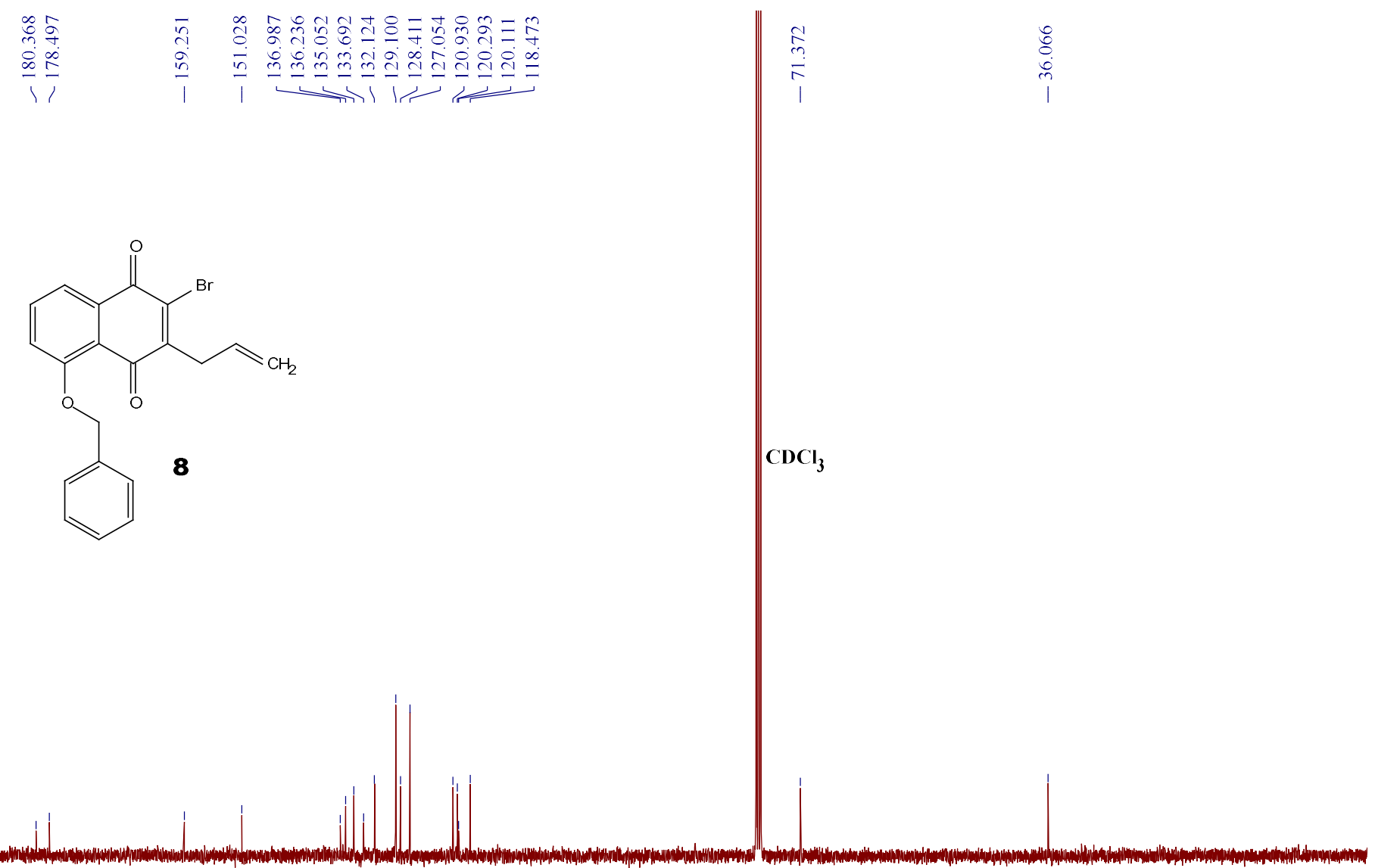


9: ${ }^{1} \mathrm{H} \mathrm{NMR}, 400 \mathrm{MHz}, \mathrm{CDCl}_{3}$

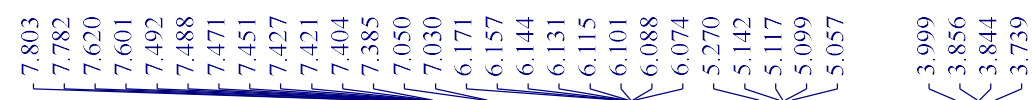

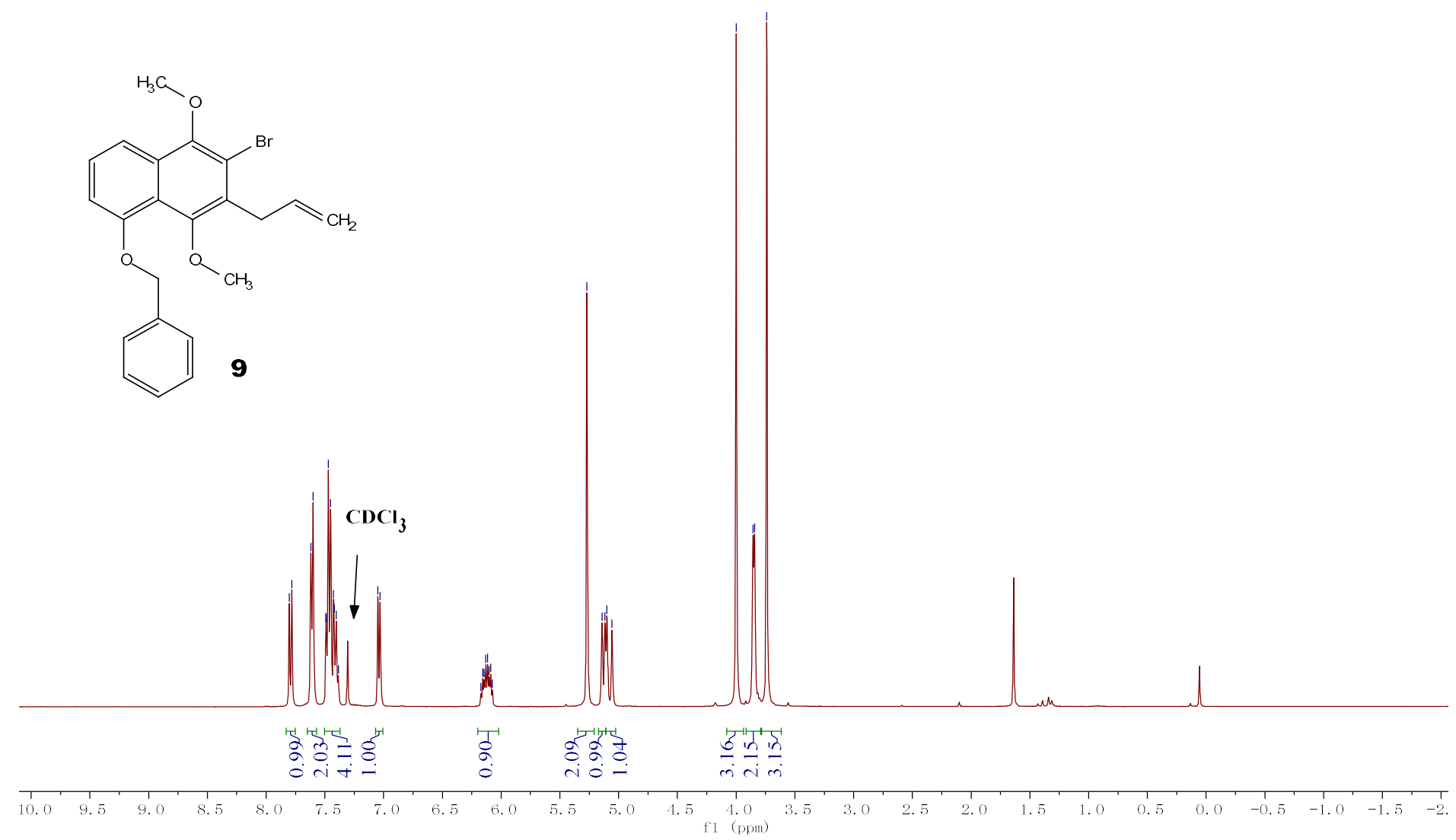


9: ${ }^{13} \mathrm{C} \mathrm{NMR}, 400 \mathrm{MHz}, \mathrm{CDCl}_{3}$

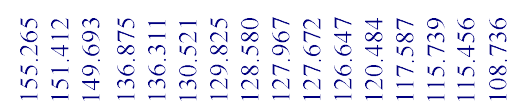

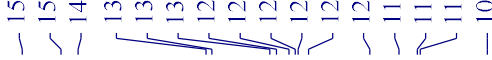
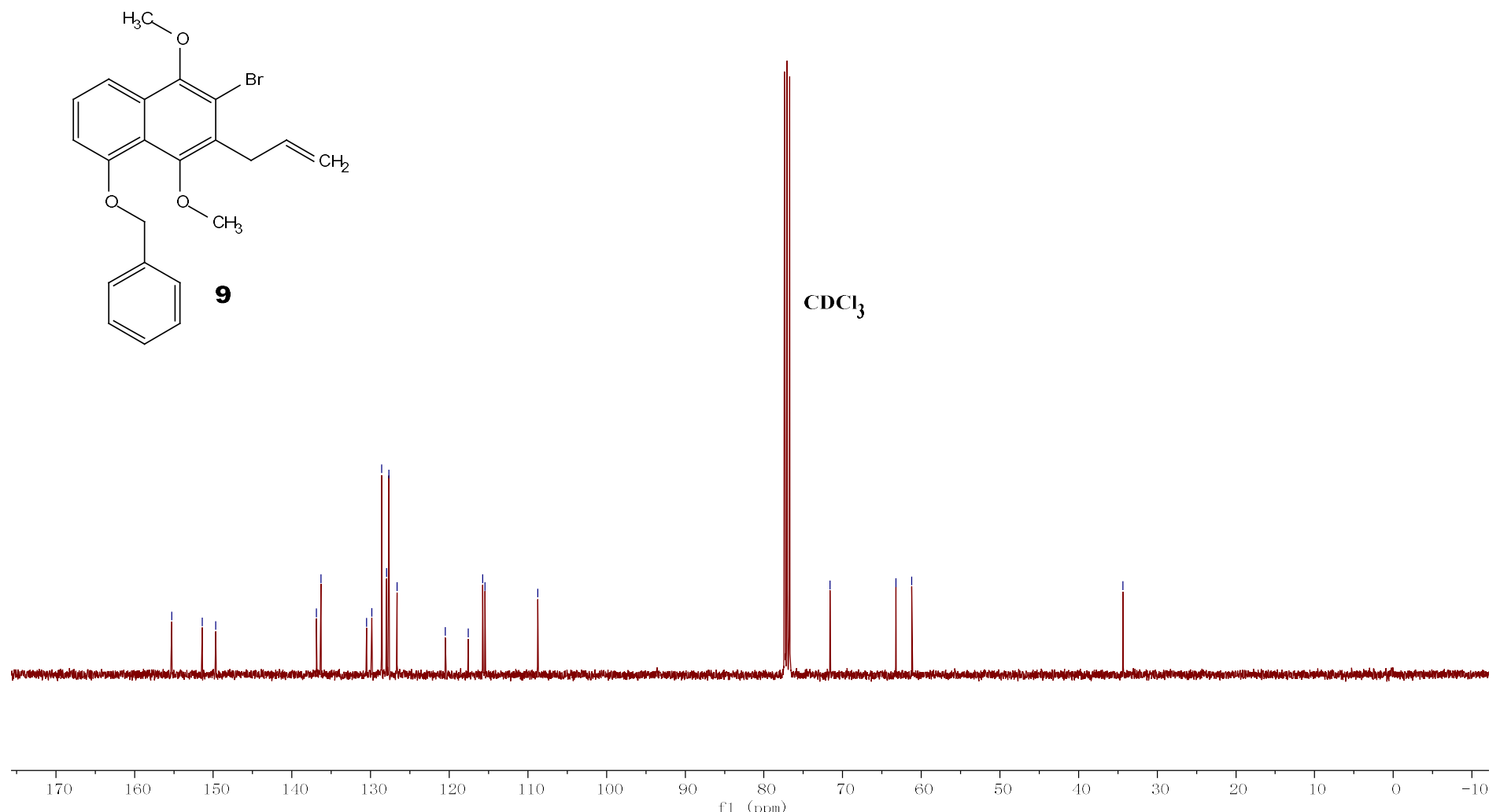
4: ${ }^{1} \mathrm{H} \mathrm{NMR}, 400 \mathrm{MHz}, \mathrm{CDCl}_{3}$
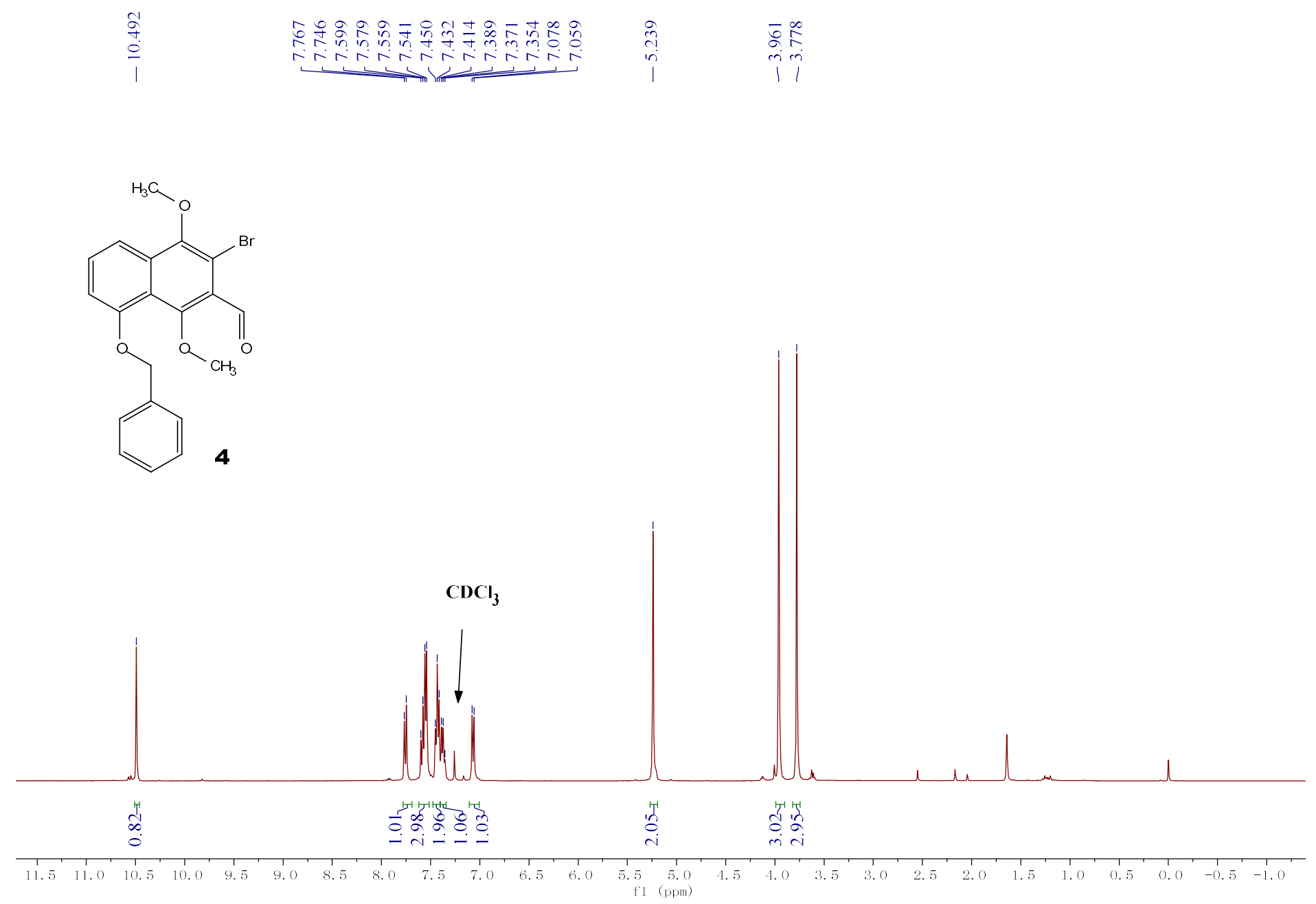
4: ${ }^{13} \mathrm{C} \mathrm{NMR}, 400 \mathrm{MHz}, \mathrm{CDCl}_{3}$
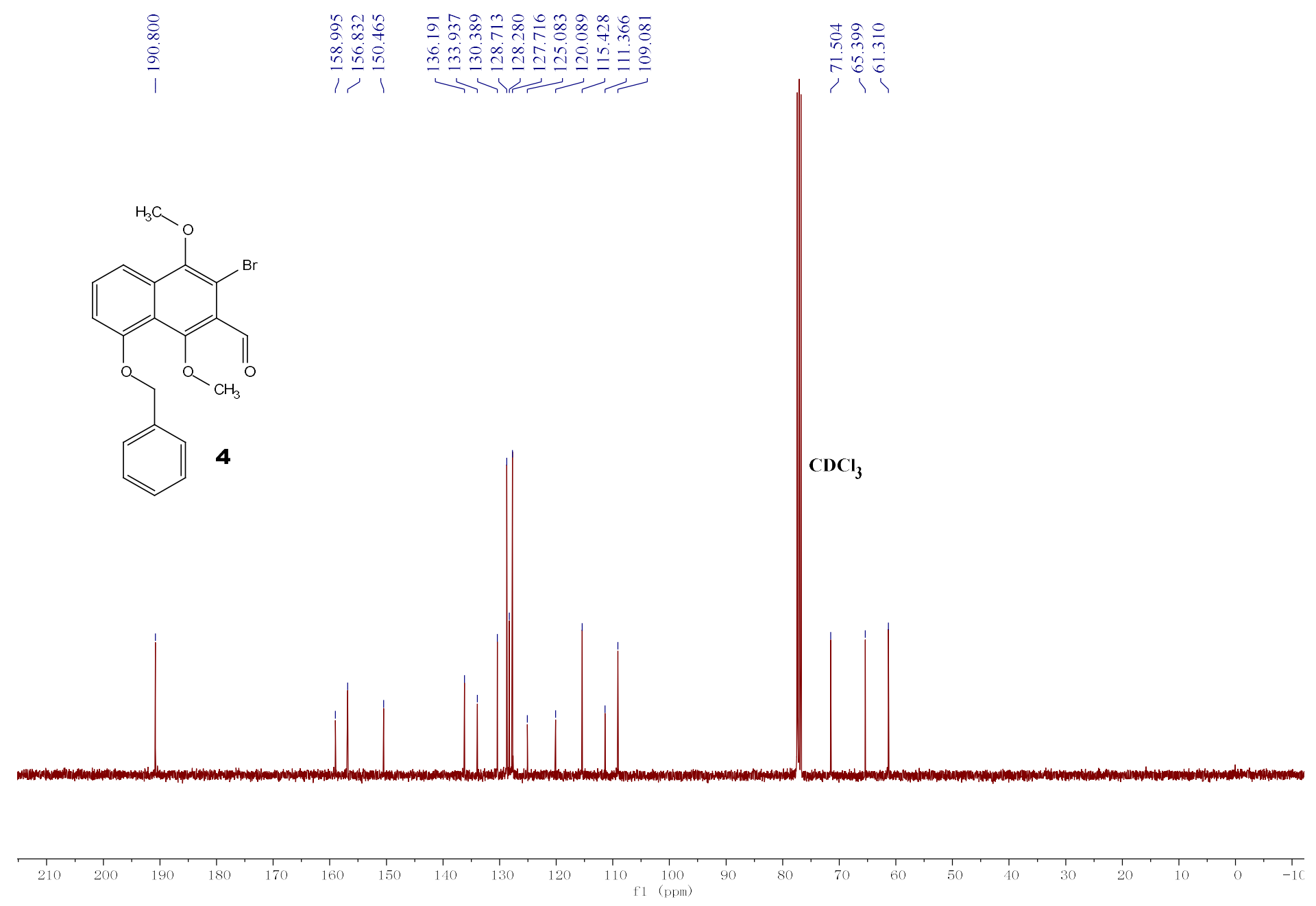

S28 
11: ${ }^{1} \mathrm{H} \mathrm{NMR}, 400 \mathrm{MHz}, \mathrm{CDCl}_{3}$

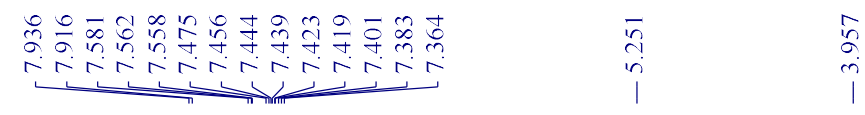

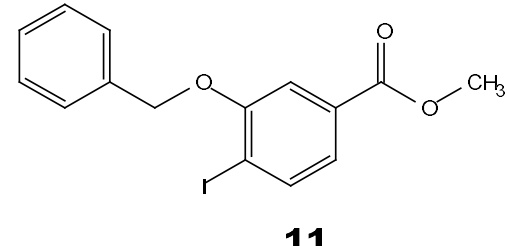

11

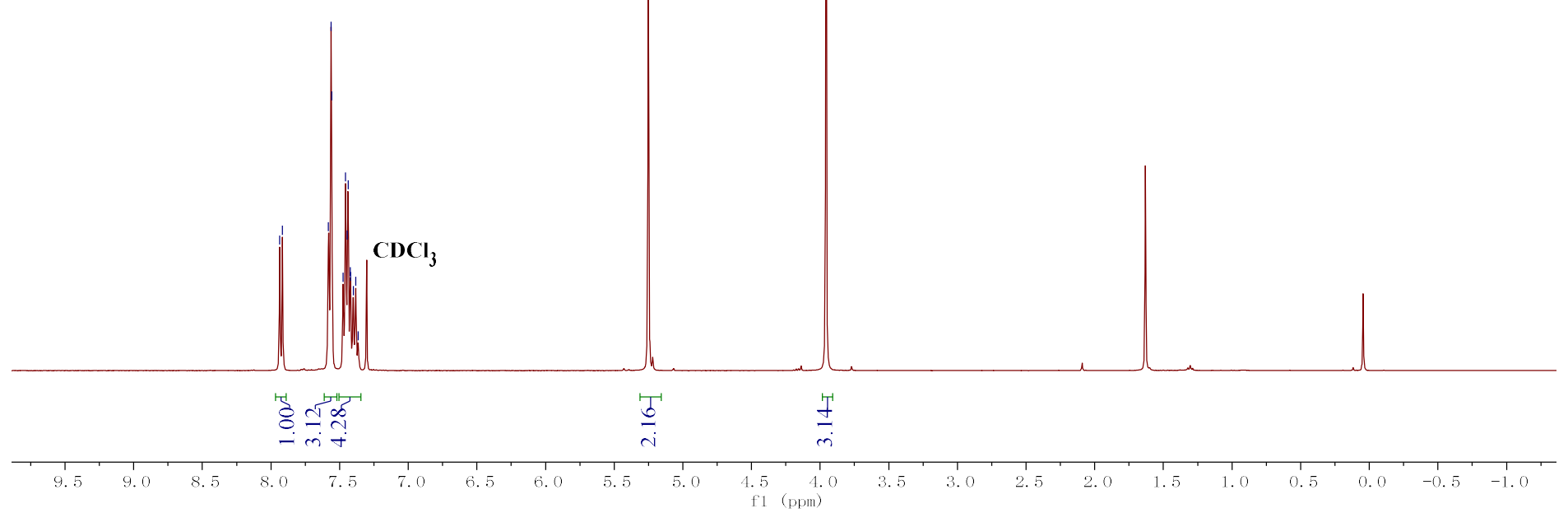


11: ${ }^{13} \mathrm{C} \mathrm{NMR}, 400 \mathrm{MHz}, \mathrm{CDCl}_{3}$
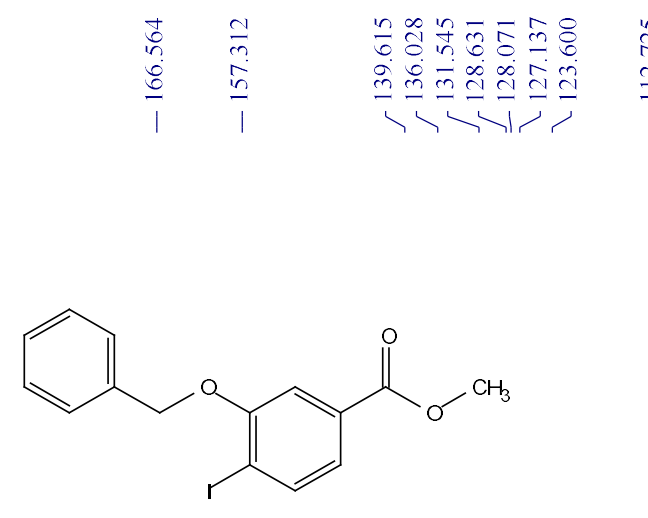

11 
5: ${ }^{1} \mathrm{H} \mathrm{NMR}, 400 \mathrm{MHz}, \mathrm{CDCl}_{3}$

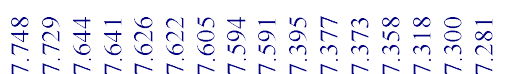

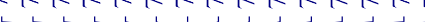
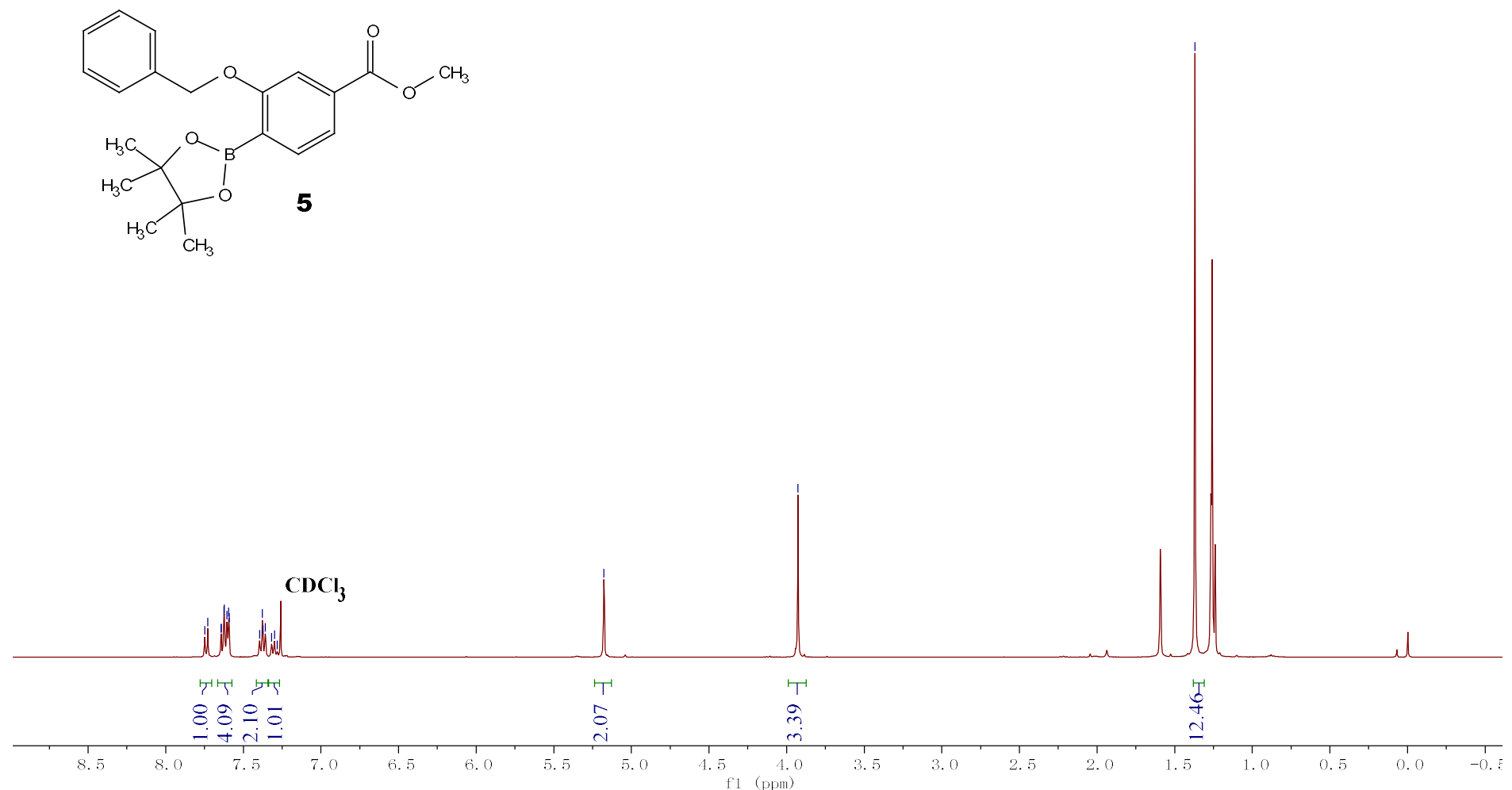
5: ${ }^{13} \mathrm{C} \mathrm{NMR,} 400 \mathrm{MHz}, \mathrm{CDCl}_{3}$
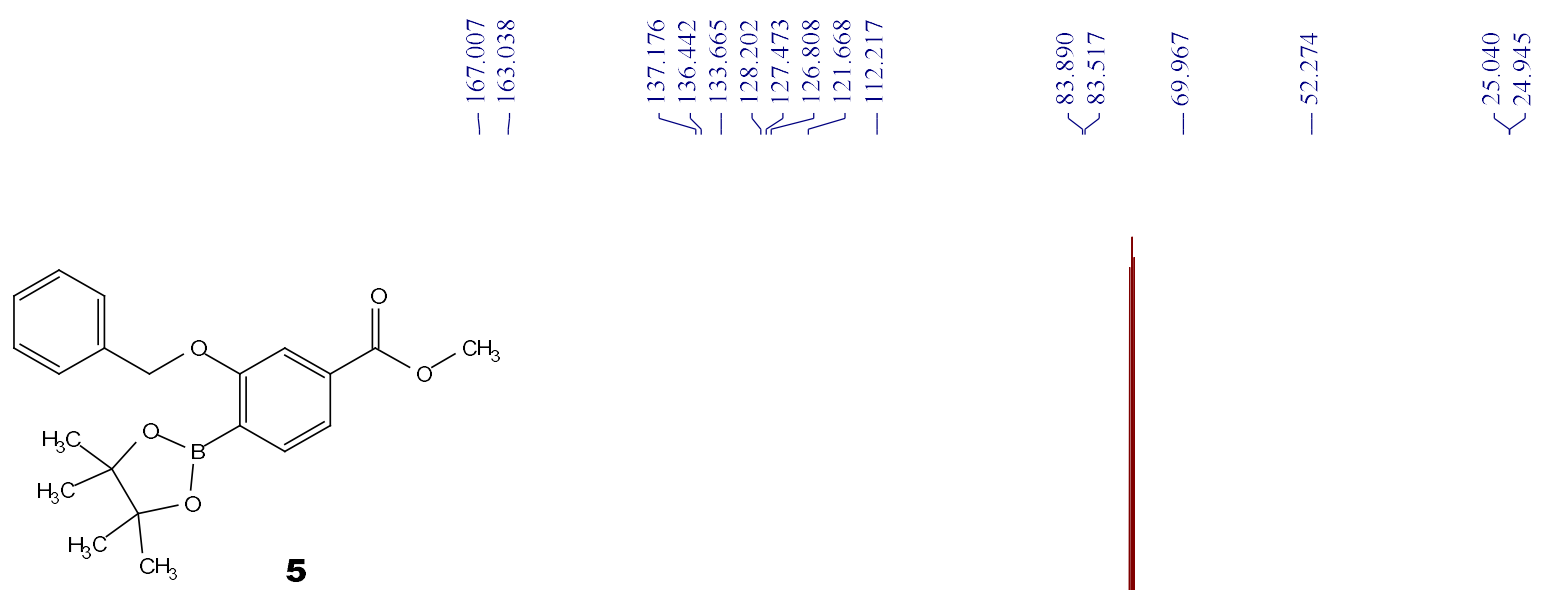

$\mathrm{CDCl}_{3}$

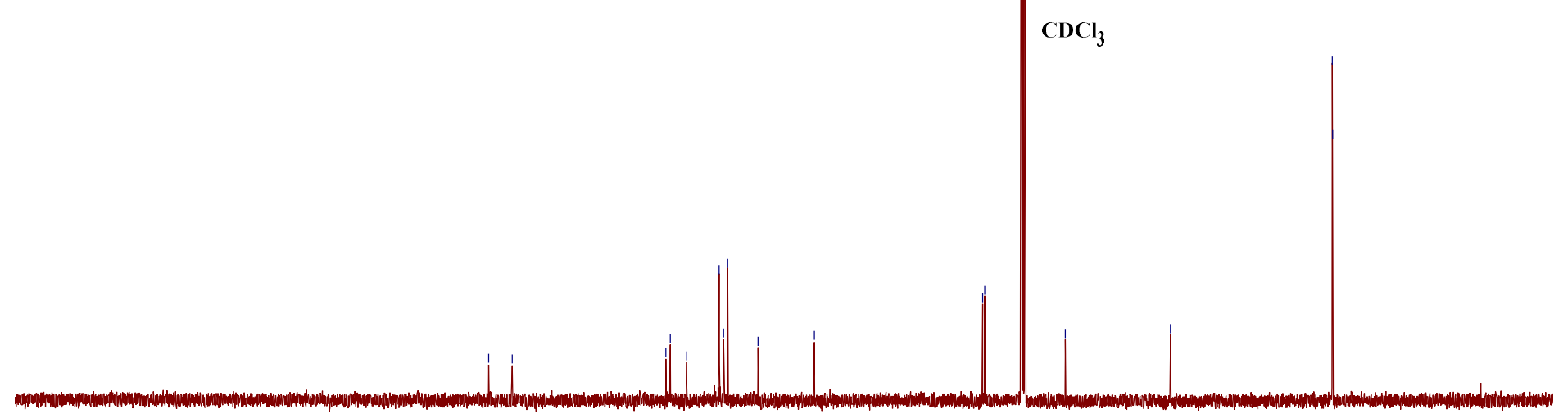


3: ${ }^{1} \mathrm{H} \mathrm{NMR}, 400 \mathrm{MHz}, \mathrm{CDCl}_{3}$

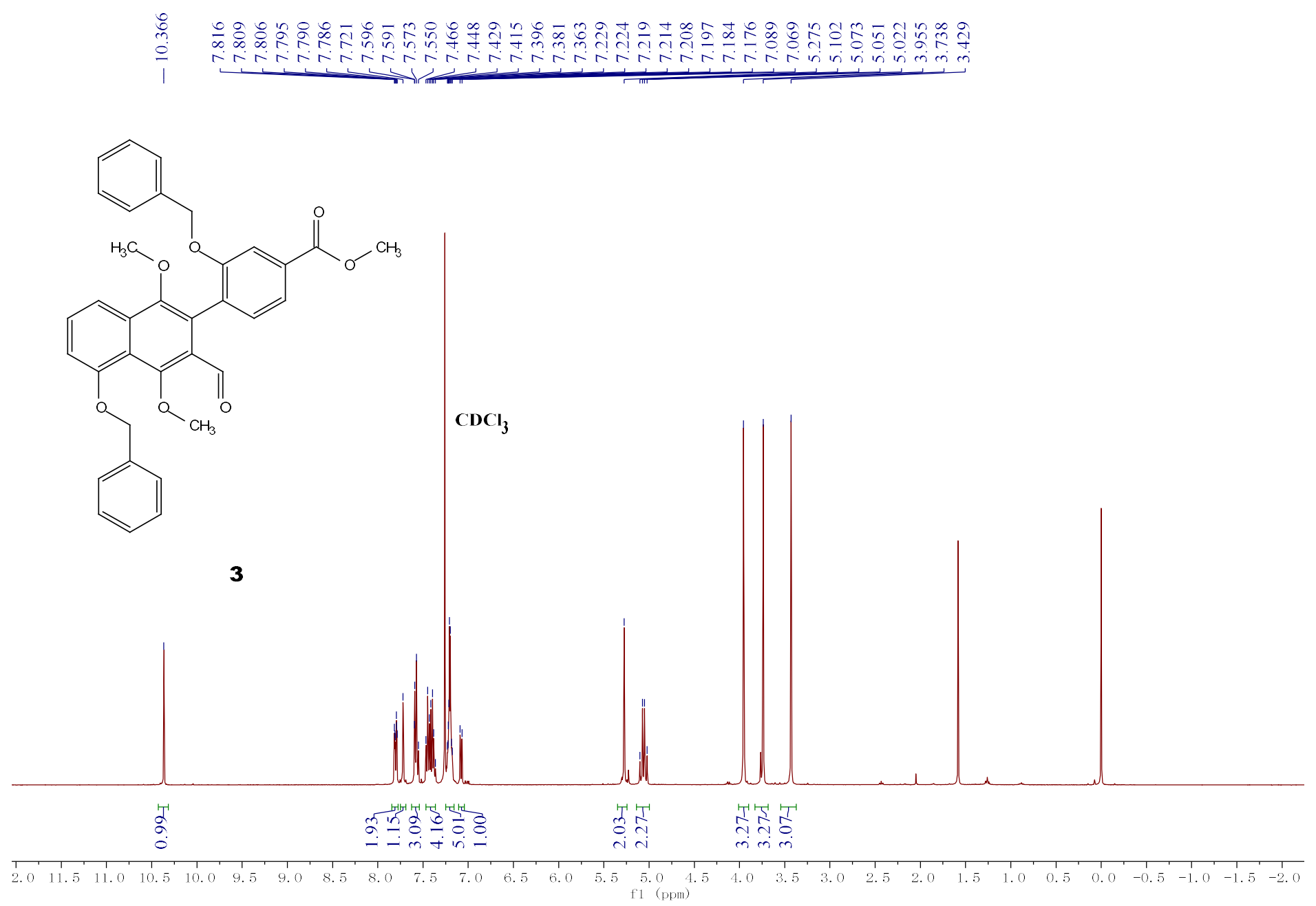


3: ${ }^{13} \mathrm{C} \mathrm{NMR}, 400 \mathrm{MHz}, \mathrm{CDCl}_{3}$

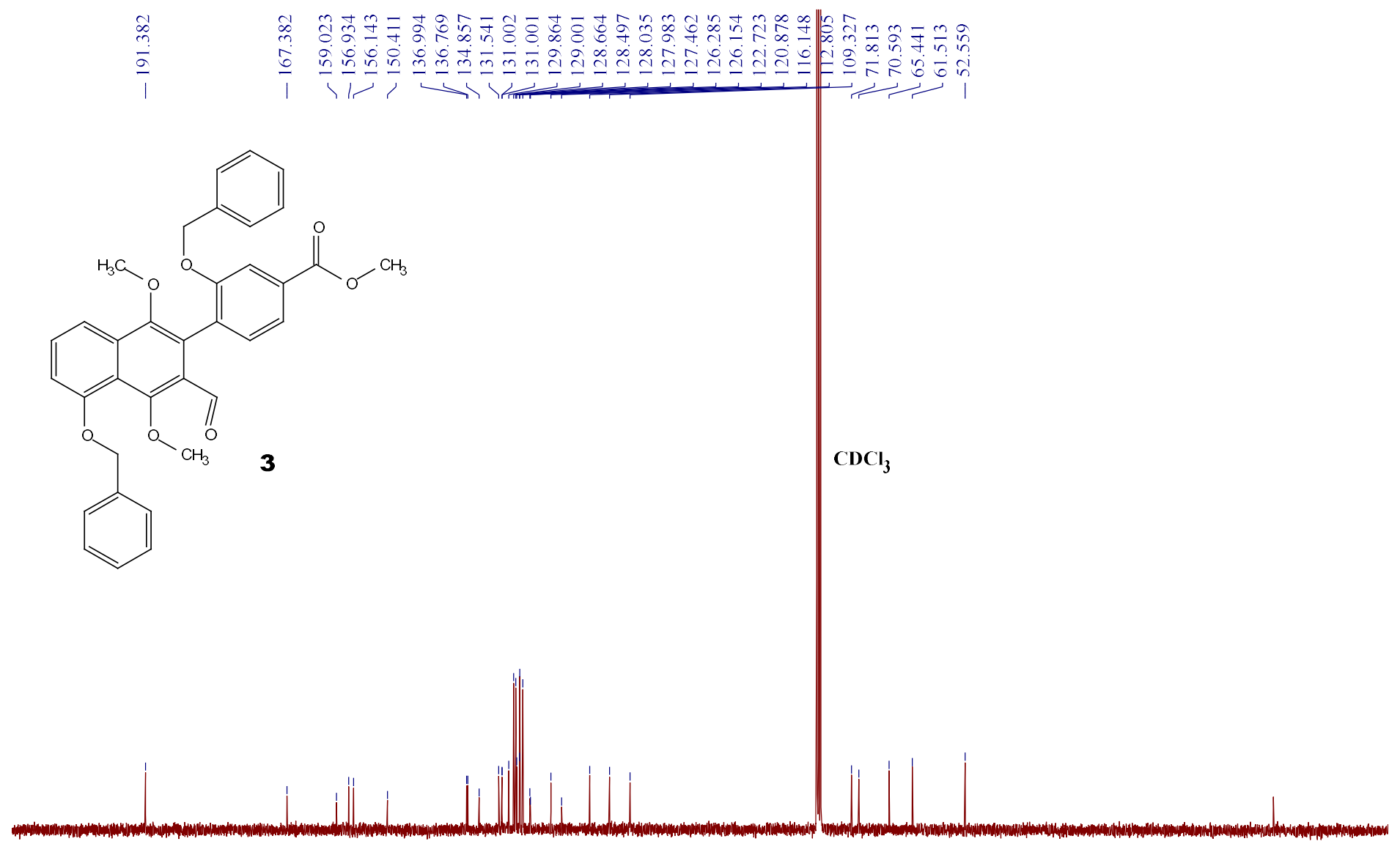


2: ${ }^{1} \mathrm{H} \mathrm{NMR}, 400 \mathrm{MHz}, \mathrm{CDCl}_{3}$
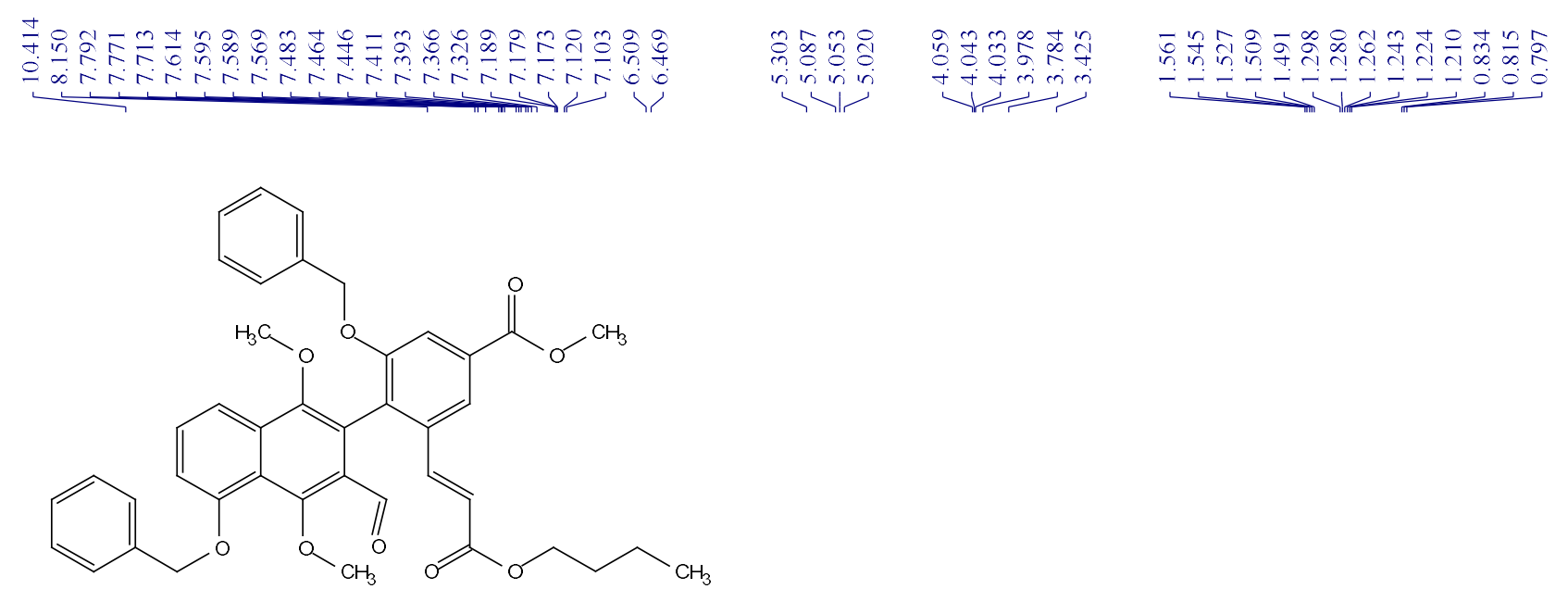

2

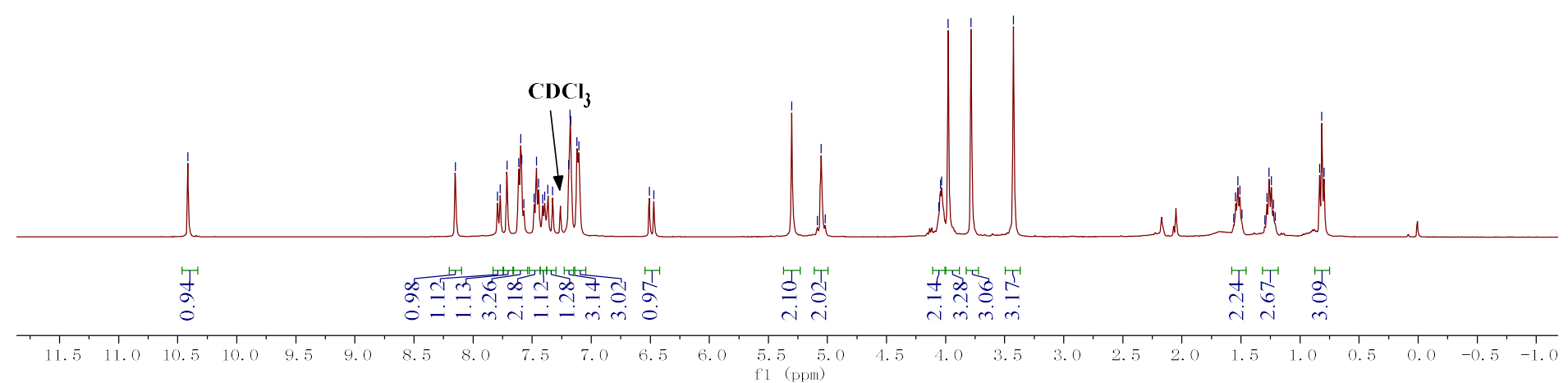


2: ${ }^{13} \mathrm{C} \mathrm{NMR,} 400 \mathrm{MHz}, \mathrm{CDCl}_{3}$
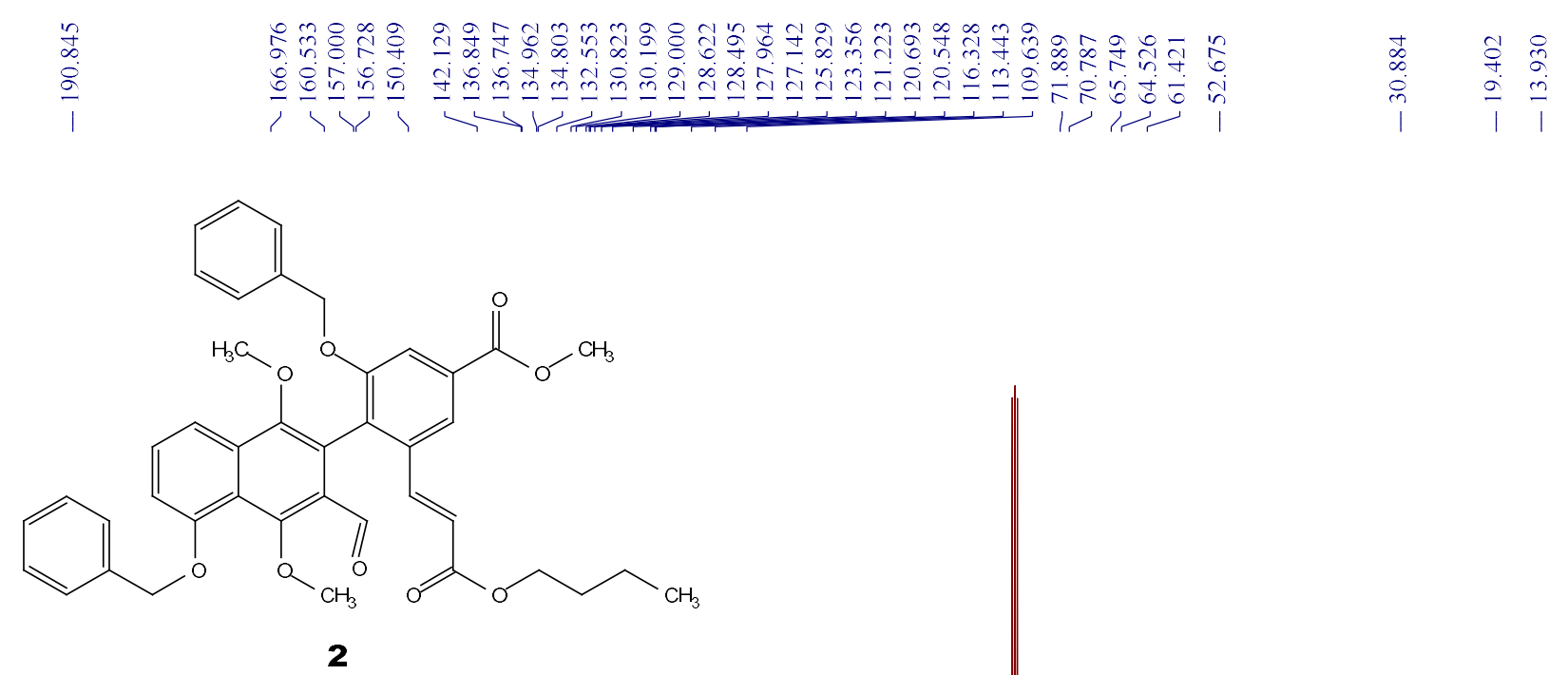

$\mathrm{CDCl}_{3}$

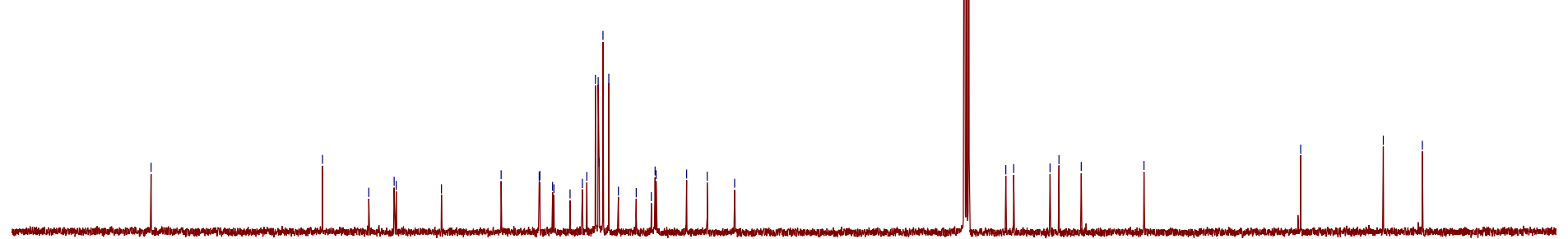

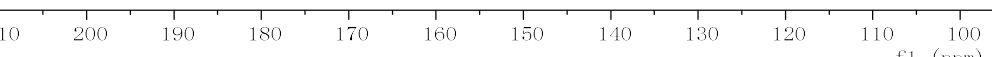


1: ${ }^{1} \mathrm{H} \mathrm{NMR}, 400 \mathrm{MHz}, \mathrm{CDCl}_{3}$
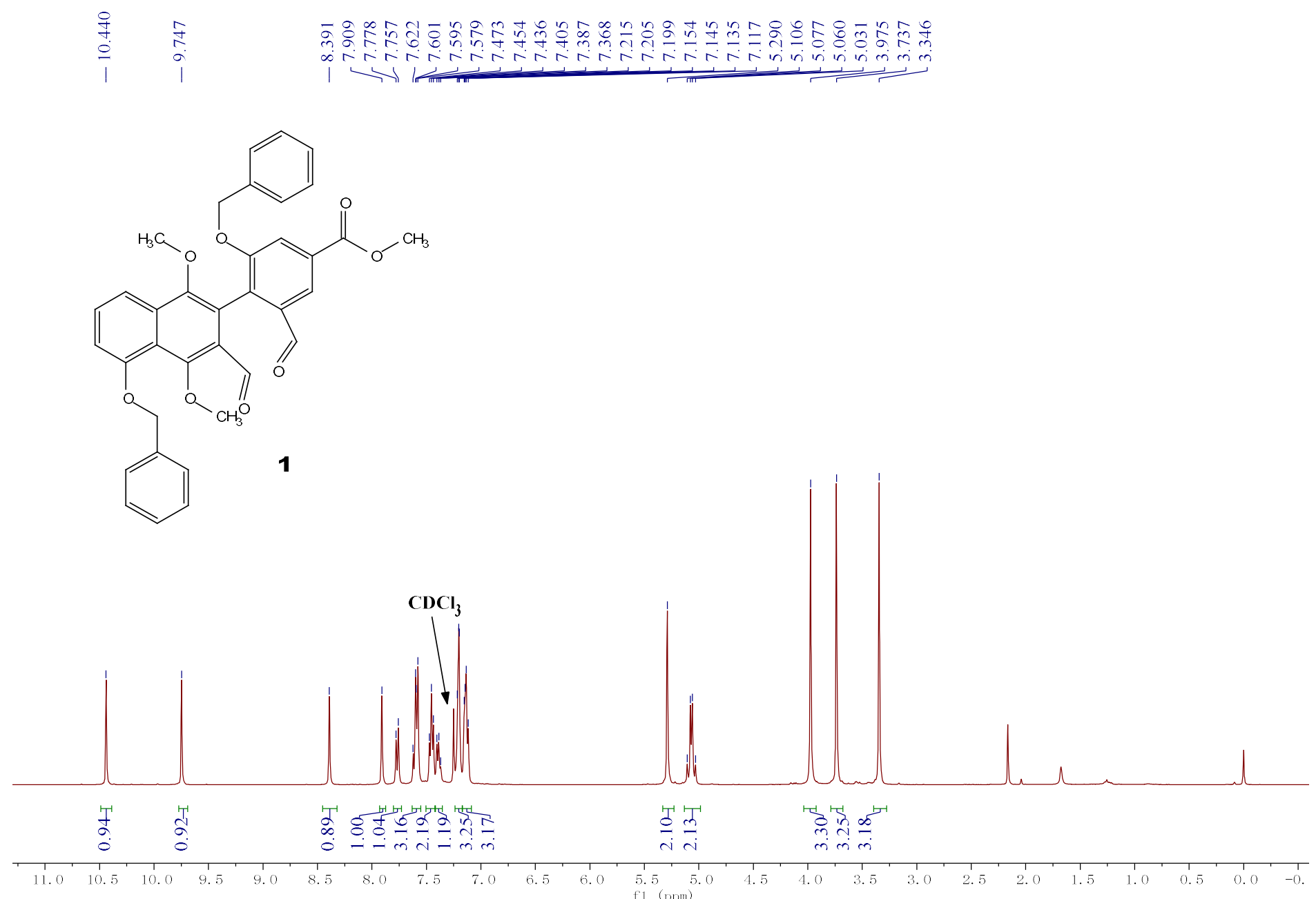
1: ${ }^{13} \mathrm{C} \mathrm{NMR}, 400 \mathrm{MHz}, \mathrm{CDCl}_{3}$

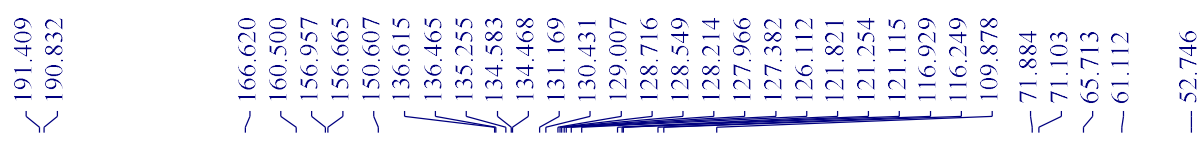
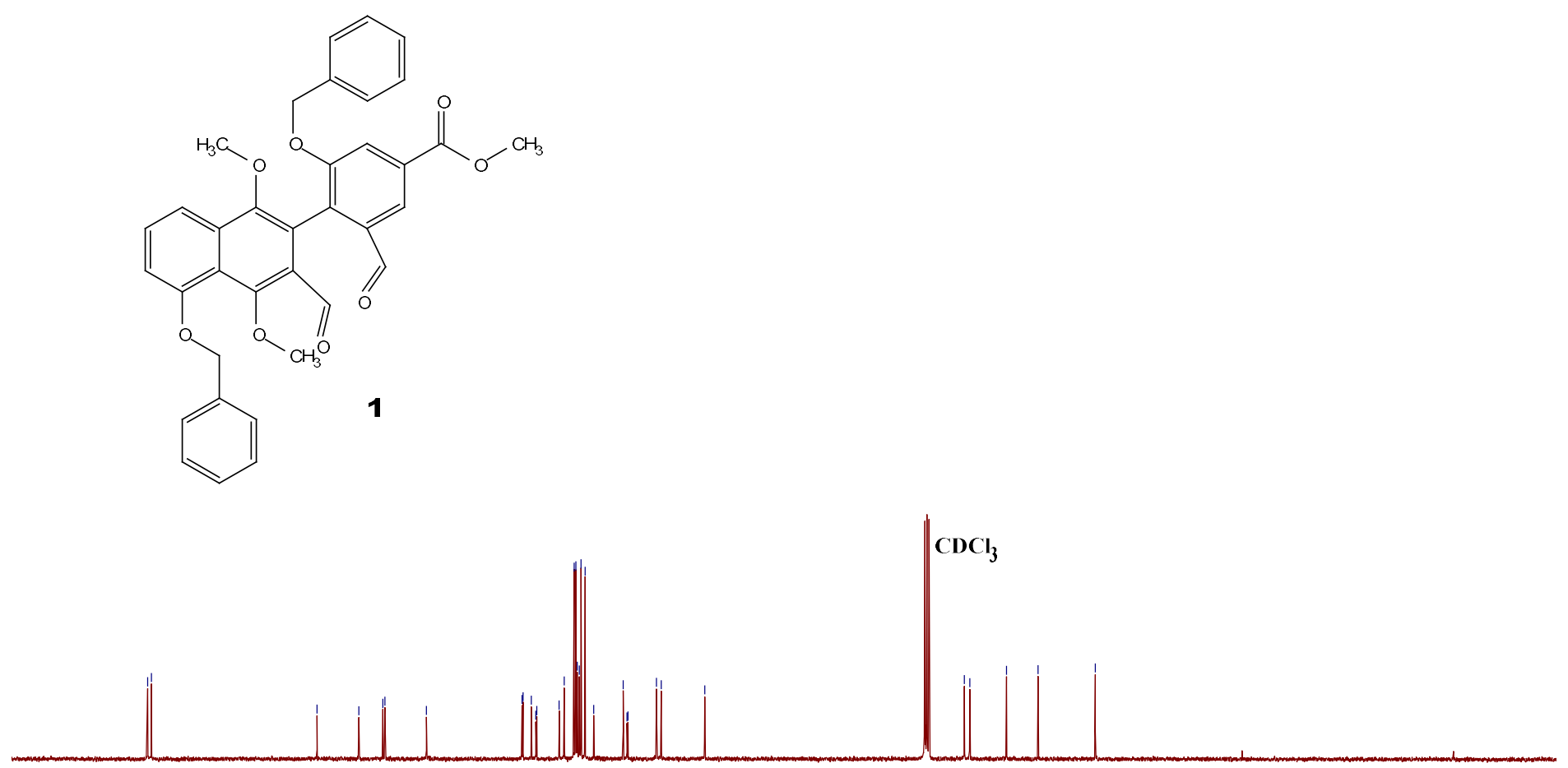

$T$

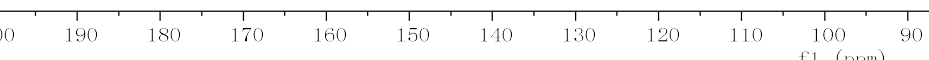


12: ${ }^{1} \mathrm{H}$ NMR, $400 \mathrm{MHz}, \mathrm{CDCl}_{3}$

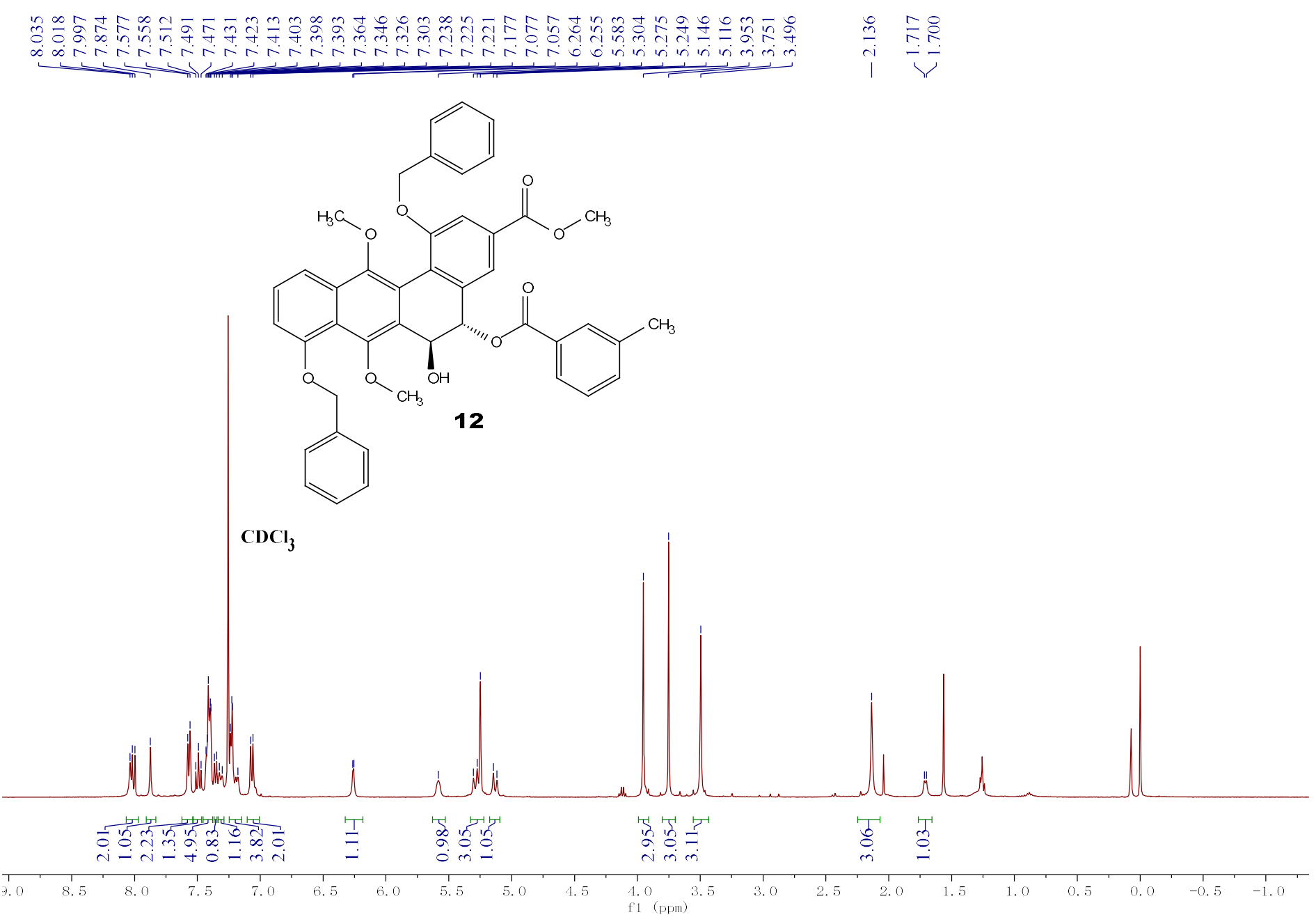


12: ${ }^{13} \mathrm{C} \mathrm{NMR,} 400 \mathrm{MHz}, \mathrm{CDCl}_{3}$

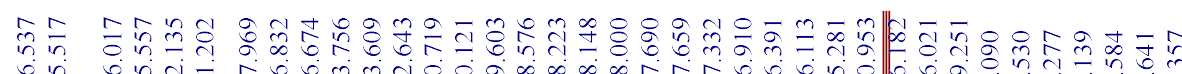

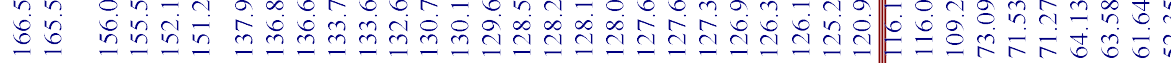

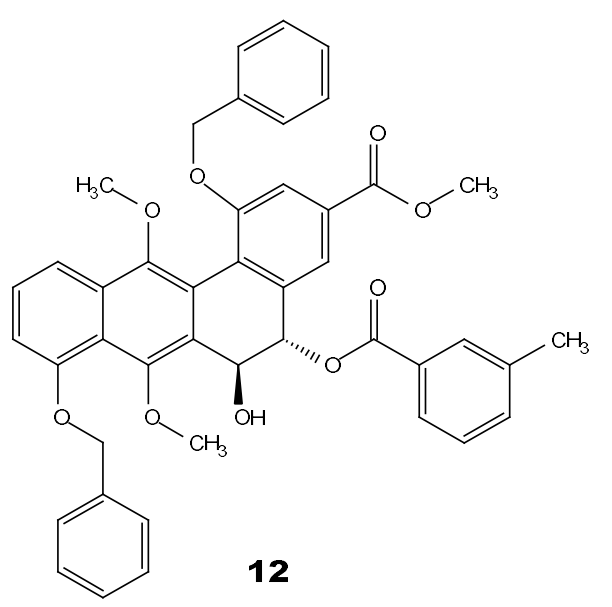

$\mathrm{CDCl}_{3}$

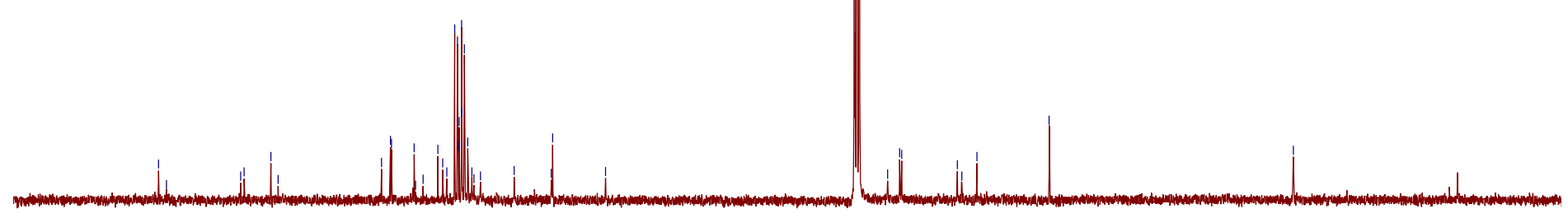

$9 \mathrm{C}$
$\mathrm{fl}(\mathrm{ppm})$ 
14: ${ }^{1} \mathrm{H} \mathrm{NMR}, 400 \mathrm{MHz}, \mathrm{CDCl}_{3}$

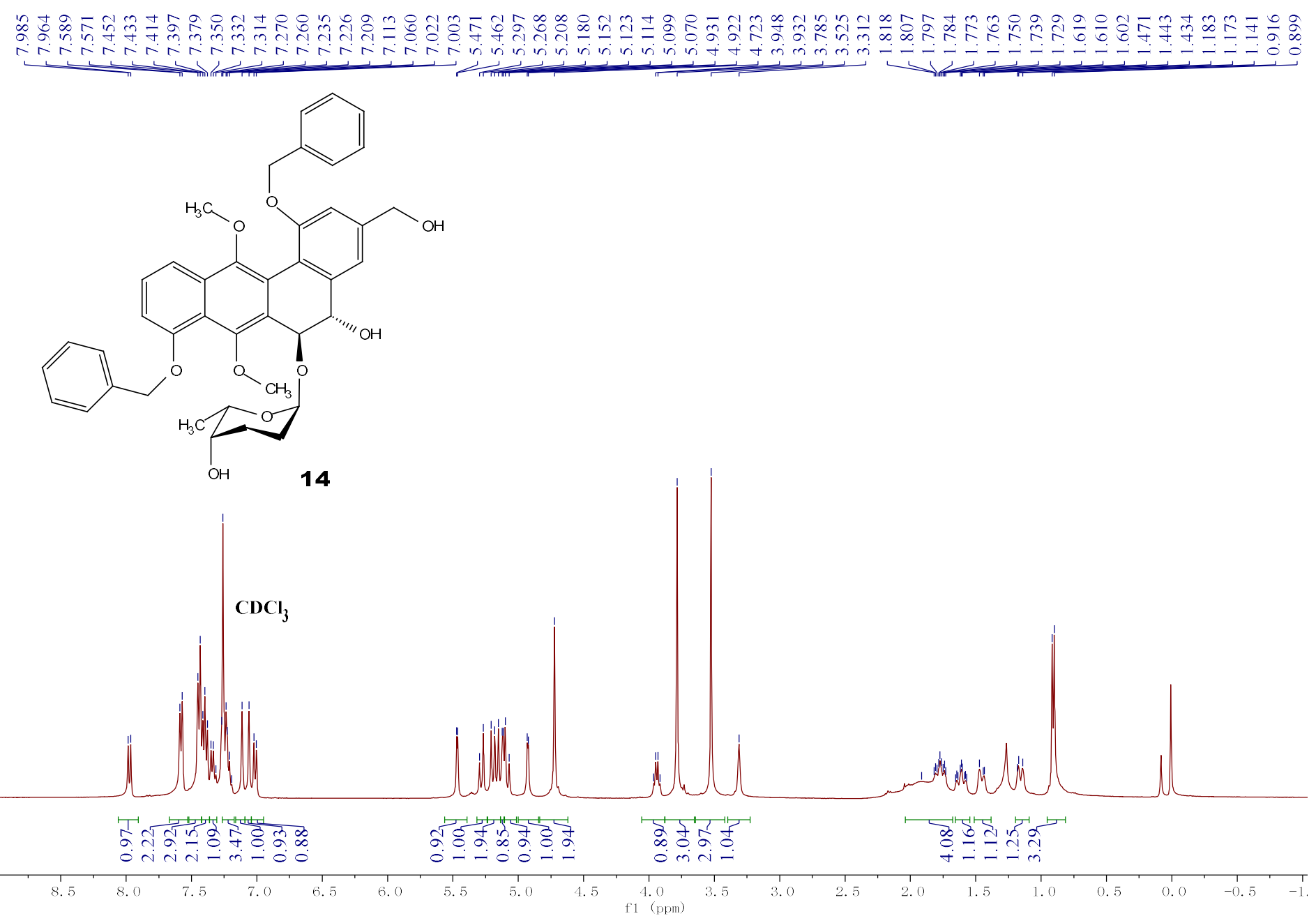


14: ${ }^{13} \mathrm{C} \mathrm{NMR,} 400 \mathrm{MHz}, \mathrm{CDCl}_{3}$

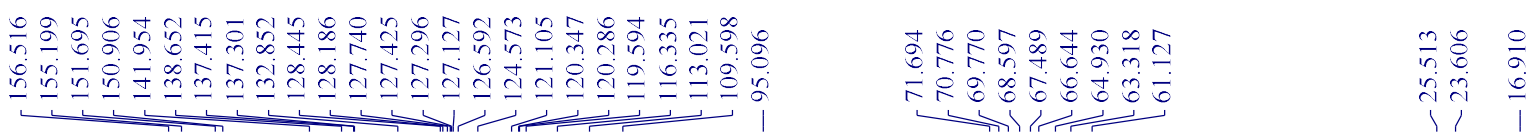
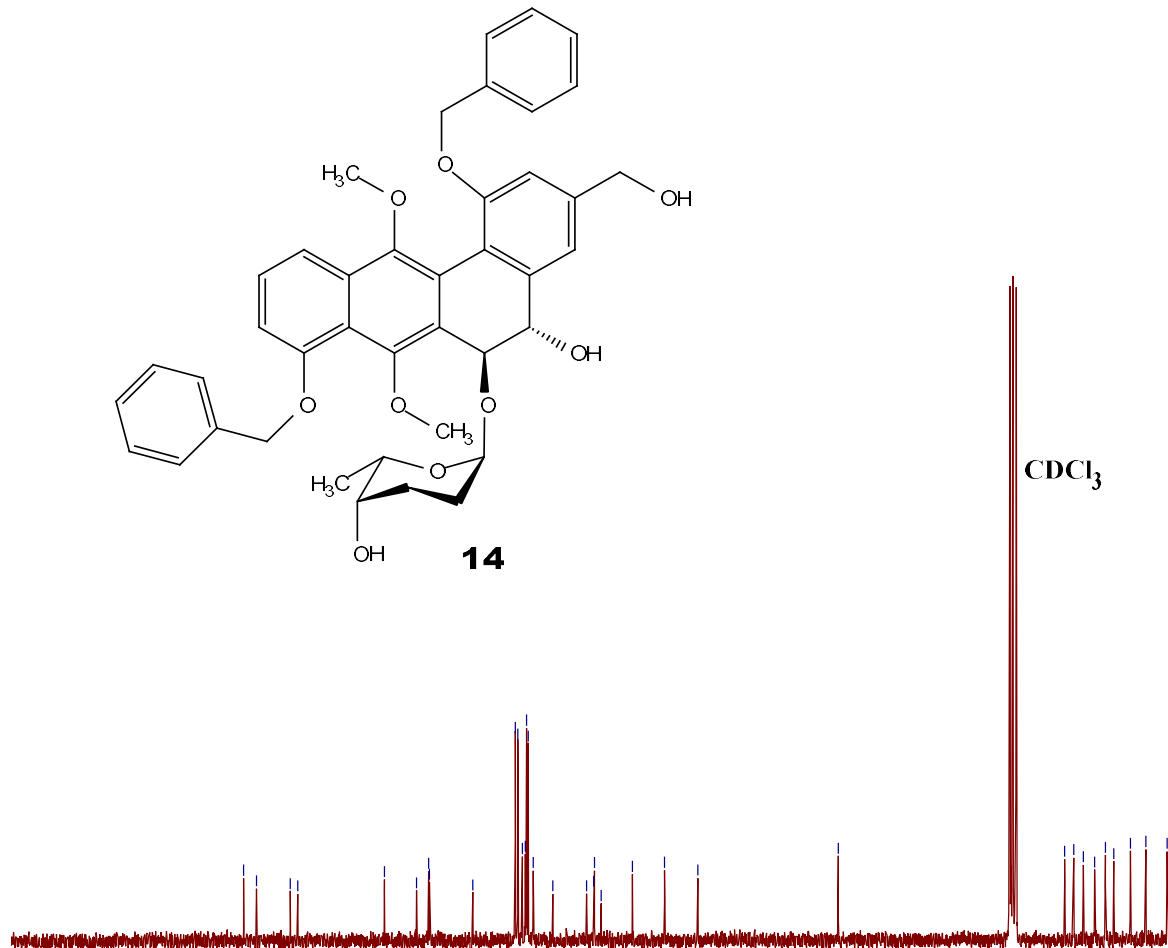

$\mathrm{DCl}_{3}$

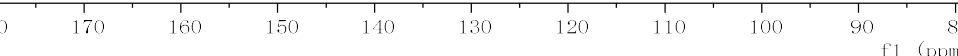


TAN-1085: ${ }^{1} \mathrm{H}$ NMR, $400 \mathrm{MHz}, \mathrm{CDCl}_{3}$

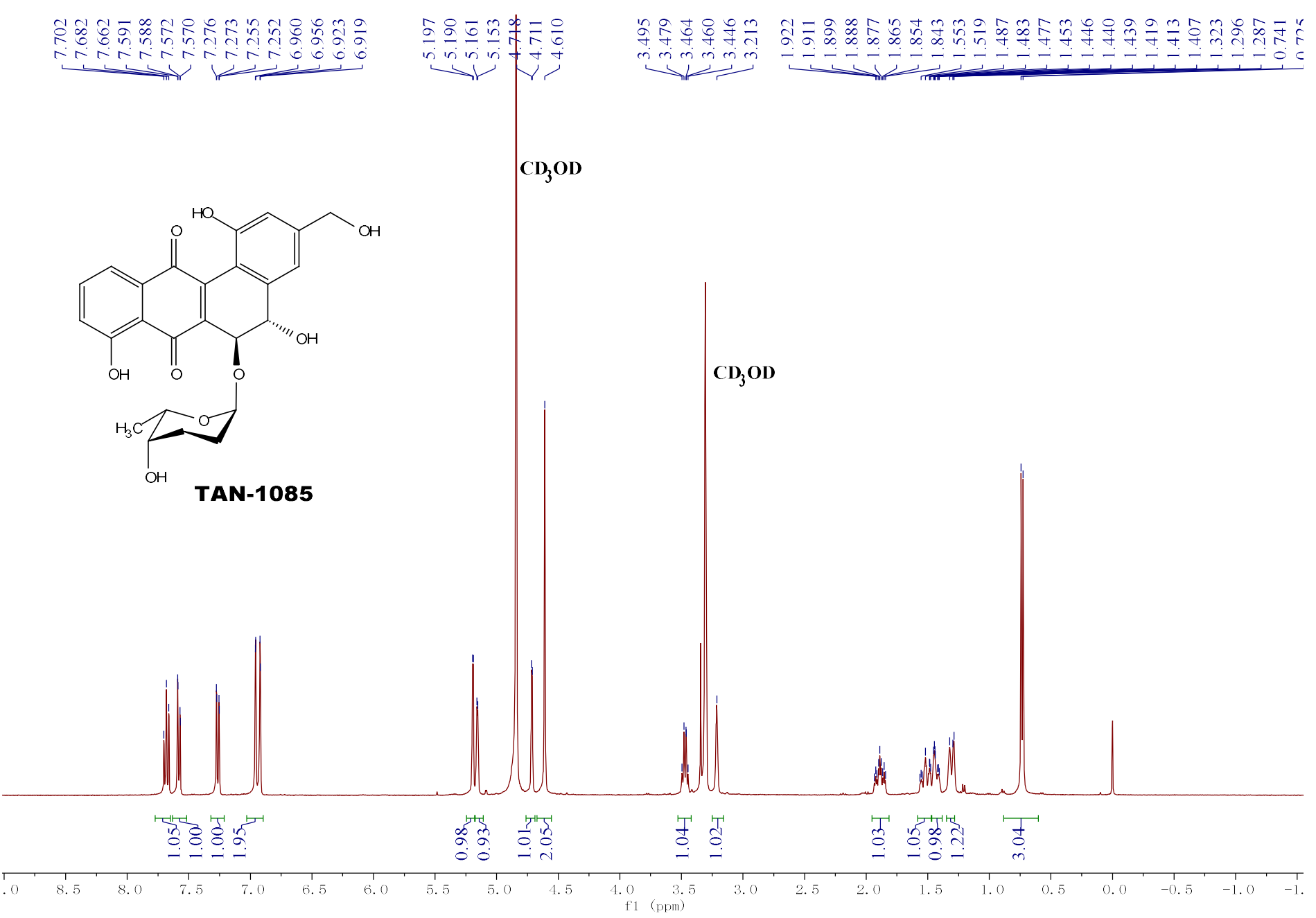


TAN-1085: ${ }^{13} \mathrm{C}$ NMR, $400 \mathrm{MHz}, \mathrm{CDCl}_{3}$

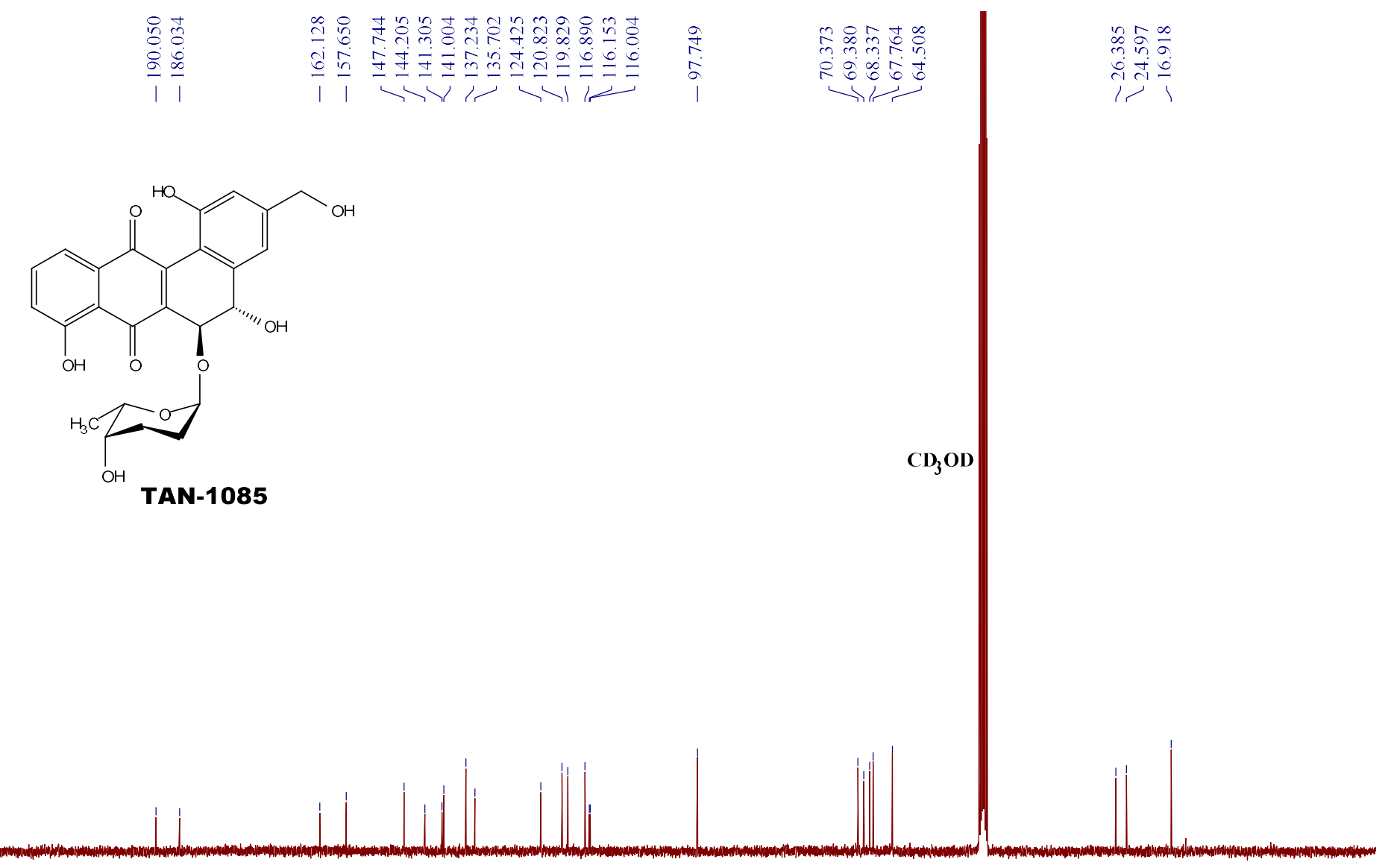

